\title{
Non-redundant functions of H2A.Z.1 and H2A.Z.2 in chromosome segregation and cell cycle progression
}

\author{
Raquel Sales-Gil ${ }^{1}$, Dorothee C Kommer ${ }^{1}\left(\mathbb{D}\right.$, Ines I de Castro ${ }^{1, \dagger}\left(\mathbb{D}\right.$, Hasnat A Amin ${ }^{1} \mathbb{D}$, \\ Veronica Vinciotti ${ }^{2, \pm}$ (D) Cristina Sisu ${ }^{1} \&$ Paola Vagnarelli, ${ }^{1, *}(\mathbb{D}$
}

\begin{abstract}
H2A.Z is a H2A-type histone variant essential for many aspects of cell biology, ranging from gene expression to genome stability. From deuterostomes, H2A.Z evolved into two paralogues, H2A.Z.1 and $H 2 A . Z .2$, that differ by only three amino acids and are encoded by different genes ( $H 2 A F Z$ and $H 2 A F V$, respectively). Despite the importance of this histone variant in development and cellular homeostasis, very little is known about the individual functions of each paralogue in mammals. Here, we have investigated the distinct roles of the two paralogues in cell cycle regulation and unveiled non-redundant functions for H2A.Z.1 and H2A.Z.2 in cell division. Our findings show that H2A.Z.1 regulates the expression of cell cycle genes such as Myc and Ki-67 and its depletion leads to a G1 arrest and cellular senescence. On the contrary, H2A.Z.2, in a transcription-independent manner, is essential for centromere integrity and sister chromatid cohesion regulation, thus playing a key role in chromosome segregation.
\end{abstract}

Keywords centromere; CPC; MYC; senescence; spindle assembly checkpoint Subject Categories Cell Cycle; Chromatin, Transcription \& Genomics DOI 10.15252/embr.202052061 | Received 9 November 2020 | Revised 26 July 2021 | Accepted 3 August 2021 | Published online 23 August 2021

EMBO Reports (2021) 22: e52061

\section{Introduction}

Nucleosomes form the basic unit of eukaryotic chromatin and consist of 146 DNA base pairs wrapped around an octamer of histone proteins. Canonical histones are incorporated into nucleosomes during DNA replication but histone variants, encoded by separate genes, are typically incorporated throughout the cell cycle (Filipescu et al, 2013; Skene \& Henikoff, 2013; Turinetto \& Giachino, 2015).

$\mathrm{H} 2 \mathrm{~A}$ is one of the four core histones. Sequence analyses have shown large-scale divergence in the $\mathrm{H} 2 \mathrm{~A}$ family, resulting in numerous variants (Bonisch \& Hake, 2012) including H2A.Z, a highly conserved variant originally identified in mouse cells (West \& Bonner, 1980). H2A.Z is present as a single variant until early deuterostomes when two H2A.Z paralogues appear: H2A.Z.1 and H2A.Z.2; they differ by only three amino acids and are encoded by the H2AFZ and H2AFV genes, respectively (Coon et al, 2005). H2A.Z.1 and H2A.Z.2 present a different L1 loop structure, and studies using fluorescence recovery after photobleaching (FRAP) showed that H2A.Z.2-containing nucleosomes are more stable than the H2A.Z.1-containing ones (Horikoshi et al, 2013). In primates, H2A.Z.2 has also two splice variants: H2A.Z.2.1 and H2A.Z.2.2, where H2A.Z.2.2 has a shorter docking domain and forms highly unstable nucleosomes (Bonisch et al, 2012).

Although H2A.Z knockdown leads to early embryonic lethality in Drosophila (van Daal \& Elgin, 1992) and mice (Faast et al, 2001), depletion of the H2A.Z orthologue in S. cerevisiae, HTZ1, is not lethal (Jackson \& Gorovsky, 2000), indicating a possible difference in the role of H2A.Z among species.

Several studies have highlighted the importance of H2A.Z in transcription regulation (Gevry et al, 2007; Giaimo et al, 2018; Dalvai et al, 2013; Chevillard-Briet et al, 2014; Rispal et al, 2019). However, whether H2A.Z promotes or represses transcription appears to depend on the gene, chromatin complex and posttranslational modifications of H2A.Z itself (Bargaje et al, 2012; Procida et al, 2021). In several organisms, H2A.Z peaks at transcriptional start sites (TSS) of active and repressed genes (Guillemette et al, 2005; Li et al, 2005; Raisner et al, 2005; Whittle et al, 2008) and localises at the +1 nucleosomes in the direction of transcription (Bagchi et al, 2020). H2A.Z has also been linked to heterochromatin regulation: recent evidence suggests that H2A.Z and H3K9me3, a known marker for heterochromatin, can cooperate to enhance the binding of Heterochromatin Protein 1 alpha (HP1 $\alpha$ ) to chromatin in vitro (Whittle et al, 2008; Ryan \& Tremethick, 2018; Fan et al, 2004).

However, the specific contribution of each paralogue towards these quite different aspects of chromatin biology is currently not clear. Although H2A.Z.1 and H2A.Z.2 are distributed similarly in the nucleus and are subjected to comparable post-translational modifications, their 3D structure and tissue distribution appear to be quite different (Dryhurst et al, 2009; Horikoshi et al, 2013). In fact, H2A.Z.2 does not compensate for the loss of H2A.Z.1 in vivo, as H2A.Z.1 knock-out is lethal in mouse (Faast et al, 2001).

1 College of Health, Medicine and Life Science, Brunel University London, London, UK

2 College of Engineering, Design and Physical Sciences, Research Institute for Environment Health and Society, Brunel University London, London, UK

*Corresponding author. Tel: +444 1895265025; E-mail: Paola.Vagnarelli@brunel.ac.uk

†Present address: Department of Infectious Diseases, Integrative Virology, Heidelberg University Hospital, Heidelberg, Germany

Fresent address: Department of Mathematics, University of Trento, Trento, Italy 
To date, very few studies have attempted to investigate and differentiate the specific roles of these two paralogues in vertebrates. In chicken DT40 cells, knock-out of H2A.Z.2 results in a slower cell proliferation rate compared with the wild-type and H2A.Z.1 knockout cells (Matsuda et al, 2010), while in humans, the FloatingHarbor syndrome (Greenberg et al, 2019) and malignant melanoma (Vardabasso et al, 2015) have been specifically linked to H2A.Z.2 (Greenberg et al, 2019). In addition, both variants seem to play independent roles in the transcription of genes involved in the response to neuronal activity (Dunn et al, 2017). Moreover, very recently, it was shown that the differences between H2A.Z.1 and H2A.Z.2 on transcription regulation seem to depend more on the relative level of the two paralogues rather than on their chromatin localisation (Lamaa et al, 2020). However, we are still missing a full understanding of the role of each variant in human cells.

As the importance of histone variants in genome organisation and regulation becomes more appreciated and a few studies have linked H2A.Z to cancer, it is important to investigate and clarify the possible differential roles of the H2A.Z paralogues and their splice variants in cell cycle regulation in vivo. This will not only provide a better understanding of their function, but it will also reveal their relative contribution to divergent aspects of chromatin biology. In this study, we have used siRNA to specifically knockdown H2A.Z.1 or H2A.Z.2 in human cells. Our results show for the first time that H2A.Z.1 and H2A.Z.2 perform non-redundant roles during cell cycle: whereas H2A.Z.1 is a key regulator of cell cycle progression at the G1/S boundary, H2A.Z.2 controls chromosome segregation and the spindle checkpoint function at the M/G1 transition.

\section{Results}

\section{H2A.Z.2 is essential for genome stability}

The H2A.Z histone variant has been linked to several and diverse functions in cellular biology and homeostasis. However, recent work has highlighted that the two H2A.Z paralogues, H2A.Z.1 and H2A.Z.2, do not perform completely overlapping roles. We therefore set out to investigate whether a separation of functions between the two paralogues was occurring in cell cycle regulation.
To this purpose, we used RNA interference to specifically deplete each variant in HeLa cells. We conducted RNA-seq on the control, H2A.Z.1 and H2A.Z.2 siRNA-treated HeLa cells to confirm the specificity of the depletion. The data show that: (i) the siRNAs are specific for each paralogue; (ii) in HeLa cells, as in many other systems, H2A.Z.1 is expressed at a much higher level than H2A.Z.2; (iii) the removal of one form does not interfere with the expression level of the other (Fig EV1A and C, and D) or histone H2A (Fig EV1B). Since there are no antibodies that can specifically distinguish between the two paralogues, we checked that the double depletion was indeed effective in depleting both forms by immunoblotting (Fig EV1C). We also analysed the percentage of depletion contributed by each single variant siRNA against the pool of H2A.Z. As the RNA-seq data suggested, the Western blot analyses confirmed that the major pool of H2A.Z in HeLa cells is provided by the H2A.Z.1 variant (Fig EV1C).

We then moved to characterise cell division and cell cycle progression in the absence of each paralogue. H2A.Z.2-depleted cells presented a high incidence of micronuclei, small nuclei formed when a chromosome or a fragment of a chromosome fails to be incorporated into the main cell nucleus after mitosis (Fig 1A-white arrows). We quantified the percentage of cells with micronuclei (Fig 1B) and the percentage of anaphase cells with chromatin bridges or lagging chromatin (Fig EV1E) upon depletion of each variant: although both H2A.Z.1 and H2A.Z.2 depletion increased the number of micronuclei and anaphase bridges, the phenotype was much stronger in H2A.Z.2-depleted cells. This effect was also confirmed using an independent siRNA oligo against H2A.Z.2 (H2A.Z.2 \#2) (Fig 1B). As H2A.Z.1 and H2A.Z.2 only differ by three amino acids, we also asked whether a particular amino acid was crucial for H2A.Z.2 role in genome stability. To this purpose, we mutated each of the three distinct amino acids of H2A.Z.2 back to the one present in H2A.Z.1, either individually or in combination, and performed rescue experiments. The H2A.Z.2 siRNA oligo targets the mRNA 5' UTR, and therefore, we tagged with GFP the coding region of H2A.Z.2 to generate the oligo-resistant mutants. An ANOVA test detected differences between all categories ( $P$-value $3.48 \mathrm{e}-10)$. We further studied pairwise differences via chi-squared tests. While proteins harbouring a single mutation were still able to ameliorate the frequency of micronuclei (to be noted that overexpression of

Figure 1. H2A.Z.2.1 knockdown leads to genome instability.

A Representative images of HeLa cells treated with control, H2A.Z.1 or H2A.Z.2 siRNA for 72 h, fixed and stained with DAPI. White arrows point at micronuclei. Scale bar: $10 \mu \mathrm{m}$.

B Quantification of the percentage of cells with micronuclei from experiment in (A). Three biological replicates were analysed for each condition (control si $N=1,271$; H2A.Z.1 si $N=1,091 ;$ H2A.Z.2\#1 si $N=1,188$; H2A.Z.2\#2 si $N=1,193)$. The error bars represent the SD. Data sets were statistically analysed using Chi-square test. $\star * * P<0.001$

C Quantification of the percentage of cells with micronuclei from HeLa cells co-transfected with H2A.Z.2 siRNA and GFP:H2A.Z.2.1 WT with either single or double mutations. Error bars represent the SD of three biological replicates (control si $N=1,316 ; \mathrm{H} 2 \mathrm{~A} . \mathrm{Z} .2 \mathrm{si} N=1,877 ; \mathrm{H} 2 \mathrm{~A} . \mathrm{Z} .2 \mathrm{si}+\mathrm{wt} \mathrm{N}=1,973$; H2A.Z.2 si $+\mathrm{A} 14 \mathrm{~T} \mathrm{~N}=618$; H2A.Z.2 si + S38T N = 558; H2A.Z.2 si + A127V N = 649; H2A.Z.2 si + A14T/S38T N = 1,090; H2A.Z.2 si + A14T/A127V N = 1,038; H2A.Z.2 si + S38T/A127V N = 1,150). Data sets were statistically analysed using Chi-square test. ${ }^{* \star *} P<0.001$; black stars refer to the comparison with the H2A.Z.2si data, and blue stars refer to the comparison with the H2A.Z.2si + wt data; no stars = non-significant.

D Schemes of the GFP constructs used for the rescue experiments in (E) and (F). Green boxes represent the GFP, brown boxes represent the H2A.Z.2.1 isoform. Solid fill represents the WT construct whereas striped box represents the KR mutant form where the residues indicate the mutations performed. Dots represent the possible post-translational modifications in the mutated amino acids: acetylation (ac, dark blue), methylation (me, pink) and ubiquitination (ub, light blue).

E Representative images of prometaphase chromosomes from HeLa cells co-transfected with H2A.Z.2 siRNA and each of the constructs in (D) (green). Scale bar: $5 \mu$ m.

F Quantification of the percentage of cells with micronuclei from experiment (E). The error bars represent the SD of three biological replicates (control si $N=887$; H2A.Z.2 si $N=1,005 ;$ H2A.Z.2 si + wt $N=760 ;$ H2A.Z.2 si + KR N = 479). Data sets were statistically analysed using Chi-square test. *** $P<0.001 ;$ ns, not significant. Blue stars refer to the comparison with the H2A.Z.2 si + wt data. 
A

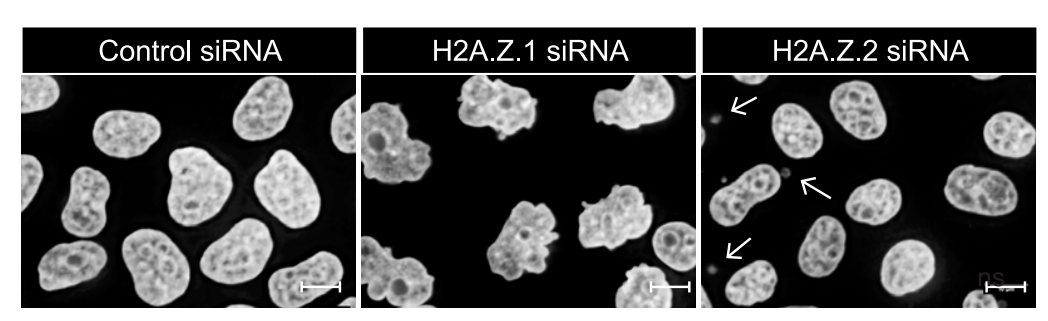

B

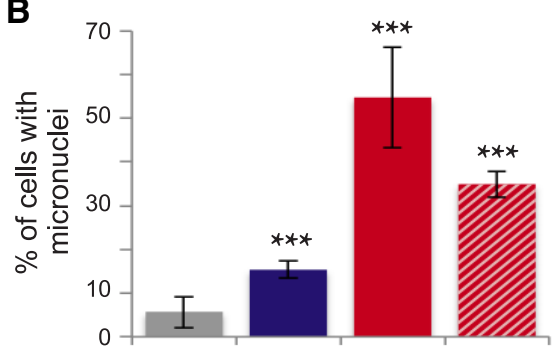

siRNA: Control H2A.Z.1H2A.Z.2 H2A.Z.2

\#1 \#2

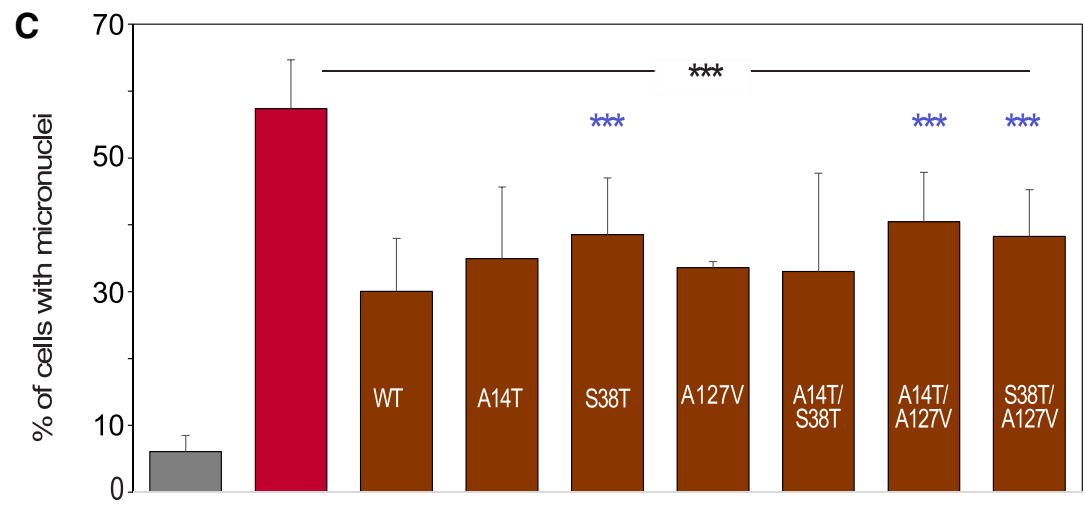

siRNA: Control

$-\mathrm{H} 2 \mathrm{~A} . \mathrm{Z} .2$

H2A.Z.2

Rescue:

\section{D}

GFP:H2A.Z.2.1wT

$:: \stackrel{\mathrm{K}^{11 \mathrm{R}}}{:}: \stackrel{:}{:}$ K4R K7R | K13R | K37R

$: \quad: \mathrm{K} 120 \mathrm{R}: \mathrm{AC}$

GFP:H2A.Z.2.1 ${ }^{\mathrm{K}}$

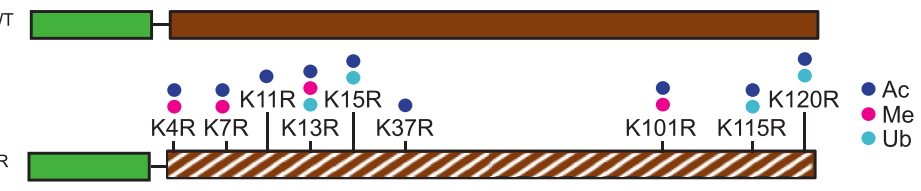

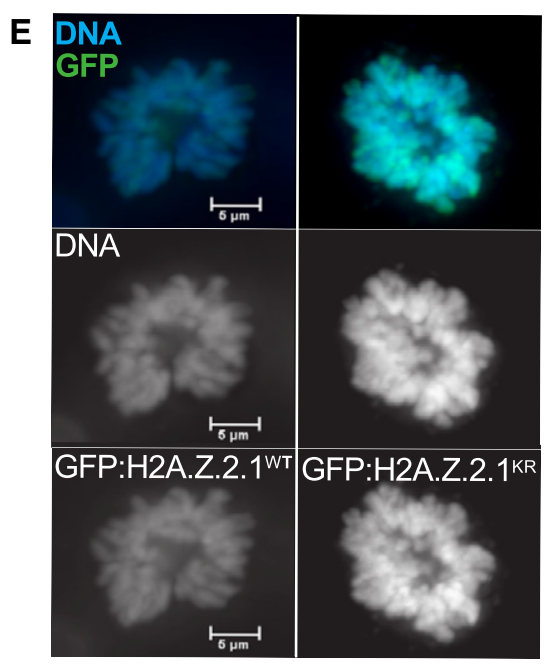

$\mathbf{F}$

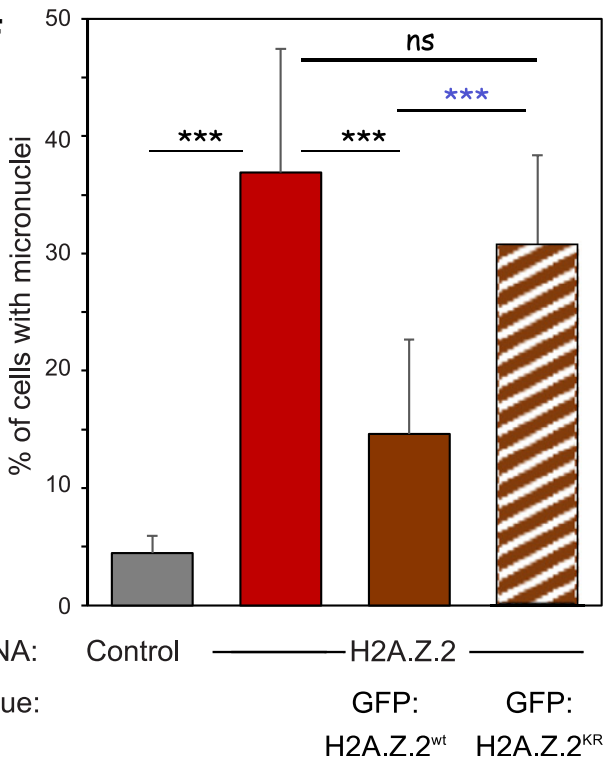

Figure 1. 
H2A.Z.2 is highly toxic to the cells; therefore, we cannot expect a full rescue of the phenotype), the extent of the rescue was still significantly different from the one provided by the WT protein for the S38T mutant (although this latter mutant was expressed at a lower level). Despite all the double mutants were expressed at the same level as the WT, only A14T/S38T was able to rescue the phenotype to a similar extent as the WT form (Figs 1C and EV1F, and Table EV1). This indicates that these few changes in amino acids have indeed a major biological impact, with A127 being one of the essential amino acids when in combination with an additional mutation.

The acetylation status of H2A.Z has been shown to influence its activity (Millar et al, 2006; Halley et al, 2010; Procida et al, 2021). We therefore wanted to test the role of acetylation (or other posttranslational modifications) for the maintenance of genome stability in mammalian cells. H2A.Z.2 also presents two splice variants: H2A.Z.2.1 and H2A.Z.2.2. The latter lacks the utmost C-terminal tail but retains the extended H2A.Z acidic patch. We first mutated all the lysines that are subjected to post-translational modifications in H2A.Z.2.1 to non-acetylable residues (arginines) (GFP:H2A.Z.2) (Fig 1D indicates the mutated residues; Giaimo et al, 2019) the GFP: H2A.Z.2.1 ${ }^{\mathrm{KR}}$ mutant still localised onto the mitotic chromosomes normally (Fig 1E). To really demonstrate that GFP:H2A.Z.2.1 ${ }^{\mathrm{KR}}$ mutant is indeed incorporated into nucleosomes, we prepared mononucleosomes from HeLa cells transfected with either GFP: H2A.Z.2.1 $1^{\text {WT }}$ or GFP:H2A.Z.2.1 ${ }^{\mathrm{KR}}$, separated the histones by SDSPAGE and detected the presence of GFP by immunoblotting (Fig EV1G-I). Collectively, this evidence demonstrates that, despite the number of substitutions, the GFP:H2A.Z.2.1 ${ }^{\mathrm{KR}}$ mutant is indeed incorporated into the chromatin. However, the KR mutant construct could not efficiently rescue the micronucleation phenotype caused by H2A.Z.2 depletion (Fig 1F, Table EV2).

We then conducted rescue experiments also with the oligoresistant mutant GFP:H2A.Z.2.2 ${ }^{\text {WT }}$ and its respective KR mutant version (the same mutations were used as for the H2A.Z.2.1 apart from $\mathrm{K} 115 \mathrm{R}$ and $\mathrm{K} 120 \mathrm{R}$, as these residues are not present in H2A.Z.2.2). Contrary to H2A.Z.2.1, H2A.Z.2.2 is not enriched on chromatin (Fig EV1J). The lack of recruitment onto the chromatin is not due to its failure of binding to the chaperone. In fact, using a LacO-LacI tethering system that we established in DT40 cells (Vagnarelli et al, 2011), we confirmed that the H2A.Z chaperone RFP:LacI:YL1 (part of both the TIP60 and SRCAP complexes) was able to recruit both isoforms to the same extent (Fig EV1L and M); as it could be expected by the behaviour of this variant, GFP: H2A.Z.2.2 was not able to rescue the phenotype (Fig EV1K).

These results suggest that the H2A.Z.2.1 variant is important for genome stability maintenance playing a key role in chromosome segregation and that post-translational modifications (acetylation and/or methylation or sumoylation) are also required for this function. However, it is also possible that these changes, although they do not affect the incorporation of the variant into chromatin, they could modify other aspects of the biology of this histone variant that ultimately result in a compromised function.

\section{H2A.Z.2 regulates sister chromatid cohesion}

We next set out to understand the molecular mechanisms underlying the micronuclei formation in H2A.Z.2-depleted cells. The presence of micronuclei could be the result of either chromosome missegregation or DNA damage arising from double-stranded breaks (DSB) and the production of chromosome fragments. To test these hypotheses, we knocked down H2A.Z.2 in a stable cell line that expresses the centromeric histone variant CENP-A tagged with YFP (YFP:CENP-A) and analysed the presence of centromeric signals in the micronuclei. As shown in Fig 2A, the majority of micronuclei have at least one CENP-A signal, indicating that they possibly contain chromosomes rather than DNA fragments. We therefore inspected prometaphases of H2A.Z.2-depleted cells and noticed that some mis-aligned chromosomes had two CENP-A signals-indicating the presence of a whole chromosome-while others presented a

\section{Figure 2. H2A.Z.2 regulates chromosome segregation.}

A Quantification of micronuclei containing 0, 1 or 2 CENP-A signals in HeLa YFP:CENP-A cells transfected with control and H2A.Z.2 siRNA for 72 h. 160 micronuclei were analysed. Error bars indicate SD of three biological replicates. The image represents an example of a micronucleus containing a single CENP-A signal. The insets are enlargements of the MN. Scale bar $5 \mu \mathrm{m}$.

B Representative images of metaphases from YFP:CENP-A (green) HeLa cells treated with control (top) or H2A.Z.2 (bottom) siRNA. The white arrowheads indicate the mis-aligned chromosomes. Scale bar: $10 \mu \mathrm{m}$.

C Representative images of metaphase spreads from control (top) or H2A.Z.2 (bottom) siRNA-transfected HeLa cells after FISH with a Chr17 centromeric probe (green). The arrowheads indicate the separated sister chromatids. Scale bar: $10 \mu \mathrm{m}$.

D Representative images of HeLa cells transfected with control (top) or H2A.Z.2 (bottom) siRNA, fixed and stained for Sgo1 (green). Scale bar: $10 \mu$ m.

E Quantification of Sgo1 localisation in prometaphase cells from the experiment in (D). Error bar represents SD of two biological replicates. 35 prometaphase cells were analysed.

F Representative images of HeLa cells treated as in (D) and stained for Aurora B (green) and alpha tubulin (grey). Scale bar: $10 \mu \mathrm{m}$.

G Mitotic index of HeLa cells transfected with control (grey), H2A.Z.1 (blue) or H2A.Z.2 (red) siRNA and treated with nocodazole for 0, 2,4 or 6 h. At least 800 cells were analysed for each category. Error bar represents SD of three biological replicates. ${ }^{\star \star} P<0.01 ;{ }^{\star \star \star} P<0.001$, ns, not significant (Chi-square test).

H Left panels: Representative images of HeLa cells treated as in (D) and stained for HP1 $\alpha$ (green). Scale bar: $10 \mu \mathrm{m}$. Right panels: Representative images of HeLa cells treated as in (D) and stained for H3T3ph (green). Scale bar $5 \mu \mathrm{m}$.

I Violin plot of centromeric H3T3ph intensity of prometaphase/metaphase cells from the experiment in (H). The median is shown as bar. Data sets were statistically analysed using the Wilcoxon rank test in $\mathrm{R} .{ }^{*} \mathrm{P}<0.01$.

J Representative images of HeLa YFP:CENP-A (green) mitotic cells after control (top) or H2A.Z.2 (bottom) siRNA treatment. Scale bar: $5 \mu$ m.

K Violin plot of centromeric CENP-A intensity of prometaphase/metaphase cells from the experiments in J. (control si $N=197, \mathrm{H} 2 \mathrm{~A} . \mathrm{Z} .2 \mathrm{si} N=260$ ). The bar represents the median. Data sets were statistically analysed using the Wilcoxon rank test in $\mathrm{R} .{ }^{\star \star} P<0.01$.

L Representative images of HeLa mitotic cells stained for CENP-C after control (top) or H2A.Z.2 (bottom) siRNA treatment. Scale bar: $5 \mu$ m.

M Violin plot of centromeric CENP-C intensity of prometaphase/metaphase cells from the experiments in L. (control si $N=1,083$, H2A.Z.2 si $N=686$, from 3 biological replicas). The bars represent the median. Data sets were statistically analysed using the Wilcoxon rank test in $\mathrm{R}$. ${ }^{\star \star \star} P<0.0001$. 
A
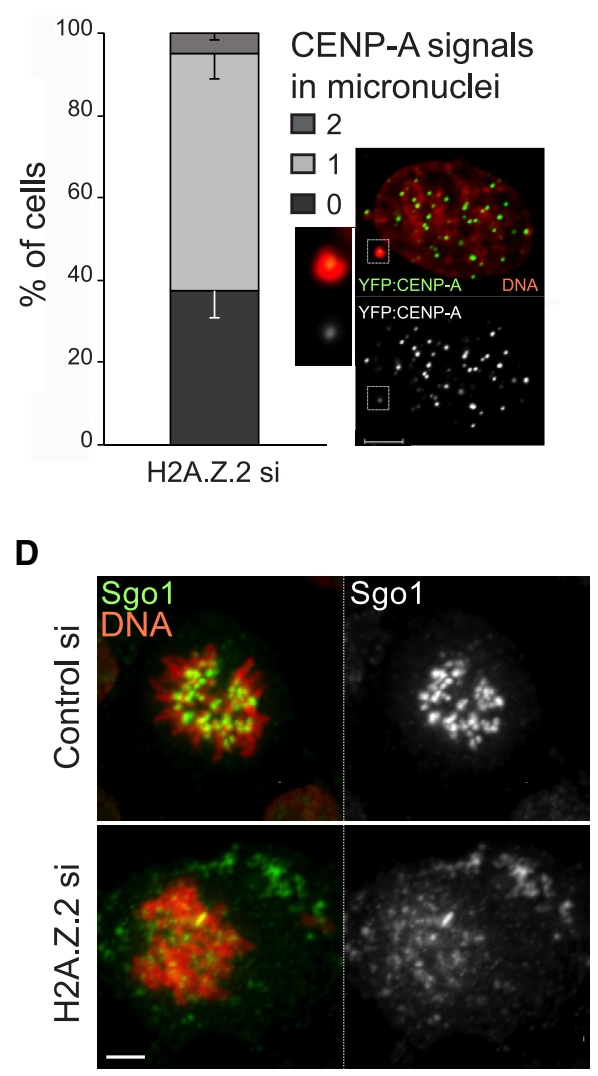

B
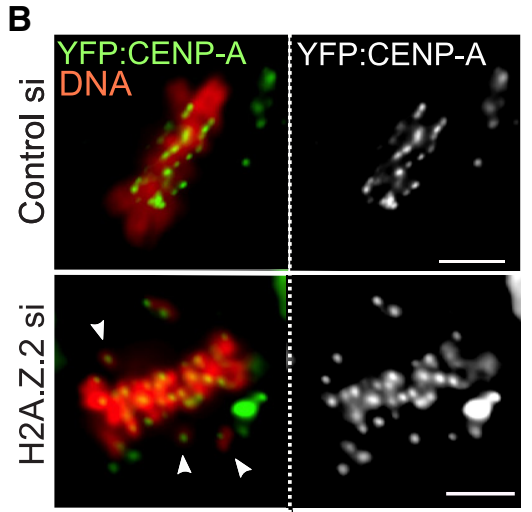

E SGO1 $\square$ On chromosomes

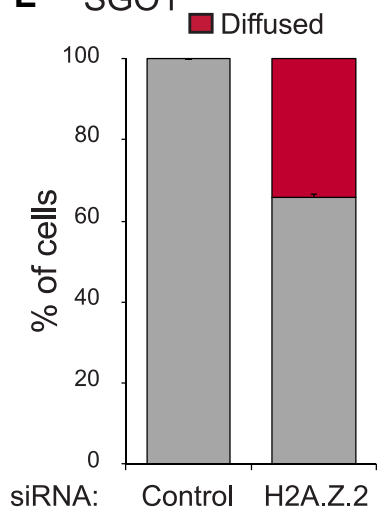

C

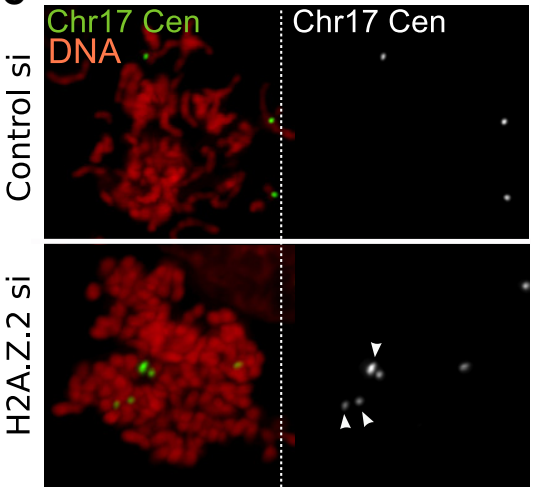

$\mathbf{F}$

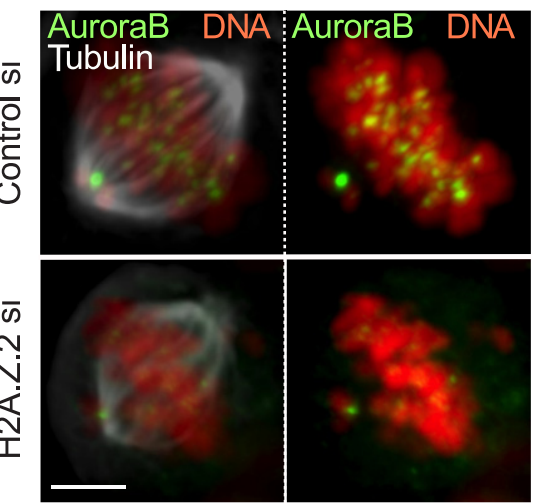

G

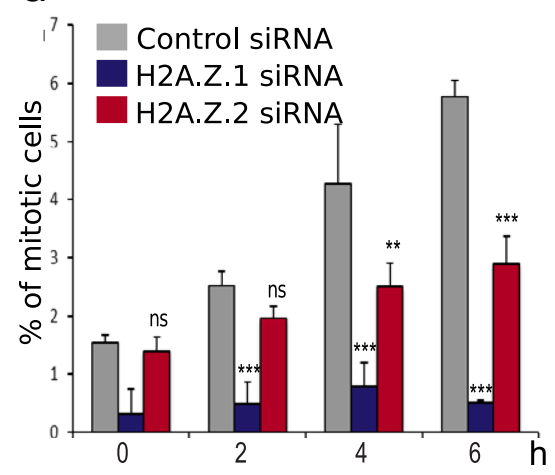

H
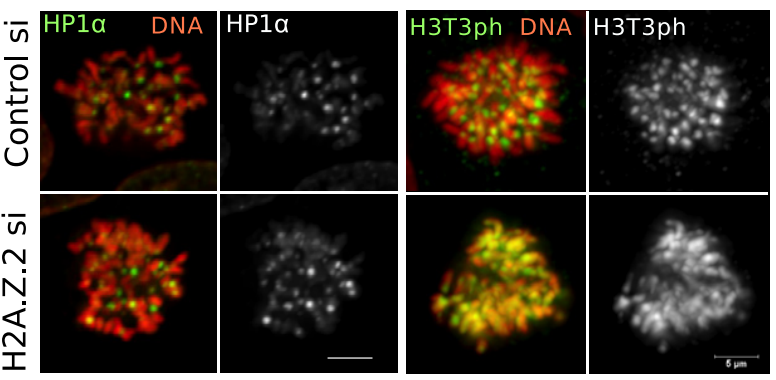

I

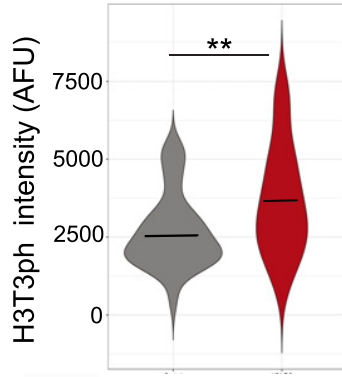

siRNA: Control H2A.Z.2

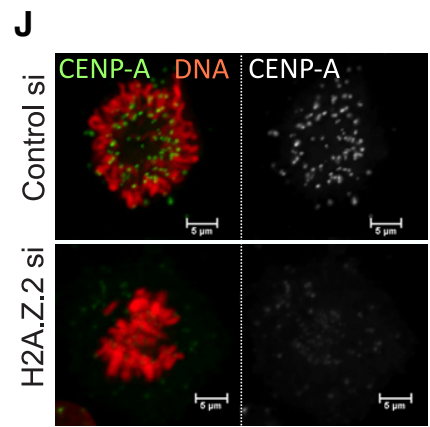

$K_{400}$

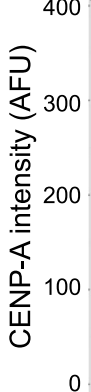

SiRNA:
$\mathbf{L}$

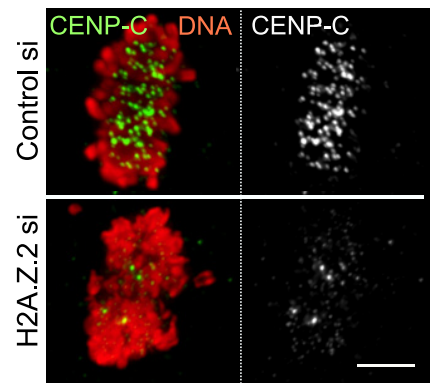

M

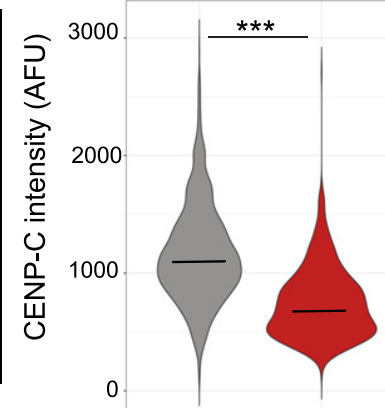

siRNA: Control H2A.Z.2

Figure 2. 
single signal-suggesting that they are single chromatids (Fig 2B, white arrows). The latter could suggest that the origin of micronuclei in H2A.Z.2-depleted cells is caused by a premature sister chromatid separation, rather than by merotelic attachments or error correction defects; this hypothesis was also supported by the analyses of mitotic chromosome spreads (Fig EV2I). In order to corroborate this observation, chromosome spreads of cells depleted for H2A.Z.2 were subjected to fluorescence in situ hybridisation (FISH) with a probe against the centromeric region of chromosome 17. In control cells, only three FISH signals are observed (the HeLa cell line used has three copies of chromosome 17) but, following H2A.Z.2 depletion, the number of FISH signals in mitosis doubled (Fig 2C); this indicates that in H2A.Z.2-depleted cells sister chromatids are prematurely separated in mitosis.

In mammalian cells, centromeric cohesin removal is prevented until anaphase onset by Shugoshin (Sgo1) (McGuinness et al, 2005). In order to investigate whether H2A.Z.2 depletion affected chromosome segregation via altering Sgol loading at centromeres, we stained H2A.Z.2- and control-depleted HeLa cells with Sgol antibodies. In $40 \%$ of prometaphase cells, Sgol failed to localise to the centromeres and appeared dispersed (Fig 2D and E). However, protein and mRNA levels of SGOL1 remained unchanged (Fig EV2A and $\mathrm{C}$ ). In a similar way, Aurora $\mathrm{B}$, that together with INCENP, survivin and borealin from the chromosomal passenger complex (CPC), was mislocalised in prometaphase/metaphase upon H2A.Z.2 depletion (Fig 2F) without any significant mRNA or protein level changes (Fig EV2B and C). Due to these results, we investigated the mitotic progression in H2A.Z.2-depleted cells, but we only found a slight decrease in the mitotic index and no significant difference in the distribution of mitotic phases (Fig EV2F-H). A compromised spindle assembly checkpoint (SAC) could be compatible with a lack of mitotic block. We therefore tested the ability of cells depleted of H2A.Z.2 to arrest upon treatment with the spindle poison nocodazole. While control siRNA-treated cells could efficiently arrest with nocodazole and the mitotic index increased over the course of the treatment, H2A.Z.2-depleted cells could not (Fig 2G); this indicates that the spindle assembly checkpoint is compromised and H2A.Z.2 plays a role in the SAC maintenance. HP1 $\alpha$ could represent the link between all these factors. In fact, HP1 $\alpha$ and Sgol are both involved in recruiting the CPC to the centromere (Kelly \& Cowley, 2013; Wang et al, 2010; Yamagishi et al, 2010; Abe et al, 2016; Ruppert et al, 2018) and HP1 $\alpha$ has been shown to be able to bind H2A.Zcontaining nucleosomes in vitro (Whittle et al, 2008; Ryan \& Tremethick, 2018; Fan et al, 2004). To test this hypothesis, we analysed the presence of HP1 $\alpha$ at the centromere in mitotic cells after control or H2A.Z.2 depletion. However, at this level of resolution, we could not detect any changes in the centromeric levels of HP1 $\alpha$ between the two samples (Fig $2 \mathrm{H}$ ). The other pathway that contributes to the localisation of the CPC to the centromere is the binding of survivin to the phosphorylated H3T3 (Kelly et al, 2010). We therefore analysed the level of H3T3 phosphorylation in control and H2A.Z.2depleted mitotic cells. Interestingly, we found a significant increase in the level of H3T3ph in H2A.Z-depleted chromosomes (Fig 2I) with a localisation that was not only limited to the centromeric region but also more diffused onto the chromosome arms (Fig $2 \mathrm{H}$, right panels). This suggests that H2A.Z.2 depletion leads to defects in the centromeric chromatin. The link between H2A.Z and H3T3 phosphorylation is not known, and therefore, we conclude that
H2A.Z.2 represents a novel pathway for the recruitment or maintenance of the CPC and/or Sgo1 to the centromere in mitosis.

Since published studies on the analyses of CENP-A chromatin have identified an enrichment of the H2A.Z variant in this fraction (Foltz et al, 2006), we investigated whether depletion of H2A.Z.2 would affect centromere maintenance. Analyses of CENP-A levels using the YFP:CENP-A cell line showed that CENP-A is reduced at centromeres of H2A.Z.2-depleted mitotic chromosomes (Fig 2J and $\mathrm{K})$ but CENP-A mRNA is unchanged (Fig EV2D). This observation could also explain why some micronuclei in the experiment analysed in Fig $2 \mathrm{~A}$ do not have a kinetochore signal. We therefore asked the question if other kinetochore markers were altered in H2A.Z.2depleted chromosomes. We quantified CENP-C levels at the kinetochores of control and H2A.Z.2-depleted mitotic chromosomes and observed a significant decrease in CENP-C upon H2A.Z.2 siRNA (Fig 2L and M) but not a decrease in CENP-C mRNA (Fig EV2E). This observation was also confirmed using an independent oligo targeting H2A.Z.2 (Fig EV2J). Reduced CENP-A levels could be at the basis of a dysfunctional kinetochore and lead to the decrease in Sgo1 and Aurora B; alternatively, the two pathways could be independent. To better understand this aspect, we analysed the correlation between Sgol and CENP-A levels in H2A.Z.2-depleted cells: the data show that a component of the dependency seems to correlate with CENP-A levels but they also reveal that other factors could be playing a role in the amount of Sgo1, particularly at the higher levels (Fig EV2K). We therefore propose that H2A.Z.2 could affect different pathways that ultimate lead to a compromised kinetochore function.

Altogether, the data suggest that in human cells the H2A.Z.2.1 paralogue, possibly regulated by its post-translational modification status, is necessary for the maintenance of a functional centromere and that its removal affects the recruitment of several chromosome segregation-related proteins to the centromere. This therefore represents a novel pathway, and the identification of the molecular details will represent a new direction of investigation.

\section{H2A.Z.1 and H2A.Z.2 have distinct roles in gene expression regulation}

Several studies have linked H2A.Z to transcription regulation (Gevry et al, 2007; Rispal et al, 2019; Vardabasso et al, 2015). We therefore wanted to investigate whether some of the effects we detected in genome stability could be explained by alteration of specific transcriptional pathways upon H2A.Z.2 depletion. In this context, we also wanted to compare the similarities and differences between the two paralogues in respect to gene transcription.

We conducted RNA-seq analyses in HeLa cells following H2A.Z.1 or H2A.Z.2 depletion. Our results revealed that no changes in the expression pattern of genes related to chromatin dynamics or cell cycle regulation were present in H2A.Z.2-depleted cells (Fig EV2AD), thus strongly indicating that H2A.Z.2 function in chromosome segregation is transcription independent. However, we observed a striking difference in gene expression changes between the two variants. To analyse the features of these genes, we focused on those showing high statistically significant changes in expression $(P<0.01)$ and a fold change $>2$. H2A.Z.1 depletion resulted in 147 downregulated genes and 107 upregulated genes; H2A.Z.2 depletion resulted in 90 downregulated genes and 119 upregulated genes. 
Only 5 and 13 genes were in common between the two variants among the upregulated and downregulated gene lists, respectively (Figs 3A and EV2L). Our results therefore suggest non-overlapping roles for these two paralogues in gene regulation. To investigate these differences in greater detail, we analysed the distribution of H2A.Z within the genes affected by the two paralogues depletion. Based on published ChIP-seq data sets (as no antibody is able to discriminate between the two paralogues, ChIP-seq data sets contain genomic regions occupied by either), we compared the distribution of H2A.Z at three different regions ( $\mathrm{i}-$ the gene body, ii-at transcription start sites $\pm 1 \mathrm{~kb}$ (TSS) and iii-other parts of the gene) for the whole genome and the four different groups of genes that change expression upon H2A.Z.1 or H2A.Z.2 depletion: upregulated or downregulated genes after H2A.Z.1 or H2A.Z.2 depletion. Interestingly, H2A.Z is enriched at the TSS of genes that are downregulated only after H2A.Z.1 depletion (Fig 3B). This possibly suggests that, in cycling cells, H2A.Z.1 has a more pronounced role in maintaining an open chromatin at active promoters than H2A.Z.2.

In order to assess the changes in chromatin that occur upon H2A.Z.1 and H2A.Z.2 depletion, we conducted ATAC-seq analyses. These analyses also reveal a significant difference between the effect of the two variants on chromatin: depletion of H2A.Z.1 led to considerable changes in the chromatin status of cells while depletion of H2A.Z.2 only to small variations (Fig 3E and F). Again, the overlaps between the two variants depletion show distinct patterns for each variant (Fig EV2M), thus indicating that they do play distinct roles in chromatin biology. Using STRING analyses, we identified a highly significant protein-protein interaction (PPI) enrichment $\left(P=10^{-16}\right)$ only for the downregulated genes of H2A.Z.1-depleted cells. Most of these genes are linked to gene ontologies (GO) terms associated with cell division and mitosis and include MYC, MKI67 and AURKB (Fig 3C). This is in agreement with a decreased mitotic index observed in H2A.Z.1-depleted cells (Figs 2G and EV2G), suggesting that cells are not cycling. Three out of the four $\mathrm{CPC}$ components were significantly downregulated following H2A.Z.1 knockdown (Aurora B, borealin and survivin). Furthermore, analysis of ENCODE/Broad Chip-Seq data shows that
H2A.Z is indeed mainly localised at the promoters of both CPC members and cMYC (Fig 3D).

These results indicate that H2A.Z.1 in HeLa cells is involved in cell cycle progression by promoting the expression of cell cycle-related genes. Analyses of the ATAC-seq data upon H2A.Z.1 depletion revealed that indeed changes occurred in the upstream regions of both cMYC and Aurora B compared with the control RNAi (Fig 3D).

Several genes differentially expressed upon H2A.Z.1 knockdown are localised on chromosomes $6(P<0.001), 17(P<0.01)$ and 19 $(P<0.05)$ (Fig EV2P). Interestingly, chromosomes 17 and 19 are the ones with the highest content of H2A.Z bound chromatin (as identified by ChIP) (Fig EV2P-bottom panel); however, no changes in copy number were observed for chromosome 17 (Fig EV2N and O). A quite different distribution was observed for H2A.Z.2. Chromosomes occupy specific territories in the nucleus that are characteristic for each cell type and linked to gene expression (Croft et al, 1999; Finlan et al, 2008). In order to assess whether chromosome positioning could have been affected by H2A.Z.1 depletion, we analysed the localisation of chromosome 17 using a centromeric FISH probe (Fig $3 \mathrm{G}$ and $\mathrm{H}$ ): high $\mathrm{d} 2 / \mathrm{d} 1$ ratios represent signals located at the nuclear periphery, while low $\mathrm{d} 2 / \mathrm{d} 1$ ratios represent signals located towards the centre of the nucleus. The results show that following H2A.Z.1 depletion, but not H2A.Z.2, chromosome 17 position is shifted towards the nuclear periphery; this position effect could be linked to the repression of many genes on this chromosome. Alternatively, the major reorganisation of chromatin upon H2A.Z.1 RNAi, as revealed by ATAC-seq analyses, could in turn affect the nuclear positioning of chromosome territories.

We therefore conclude that H2A.Z.1 and H2A.Z.2 have distinct effects on chromatin organisation, regulate the expression of different subsets of genes and that H2A.Z.1 is specifically involved in cell cycle progression.

\section{H2A.Z.1 depletion causes $G 1$ arrest and cell senescence}

H2A.Z.1 depletion triggers the downregulation of proliferationassociated genes such as Ki-67 and Myc leading to a marked

\section{Figure 3. H2A.Z.1 regulates the expression of cell cycle genes.}

A Gene expression of three biological replicates of HeLa cells transfected with control, H2A.Z.1 or H2A.Z.2 siRNA was analysed by RNA sequencing. Venn diagram shows the number of significant $(P<0.01)$ differentially expressed genes and the overlap between each set of genes.

B Pie charts displaying the percentages of H2A.Z peaks at or nearby transcription start sites (TSS) (dark blue), within the gene body (grey) or elsewhere in the whole genome (purple) for the differentially expressed genes following H2A.Z.1 or H2A.Z.2 depletion. A 2-sample test for equality of proportions was used for the statistical analyses. ${ }^{*} P<0.05$; ns, not significant.

C Downregulated genes following H2A.Z.1 depletion were analysed by STRING. The image shows the cluster of genes with Gene Ontology (GO) terms related to cell cycle. PPI, protein-protein interaction.

D IGV analyses of H2A.Z localisation (from ENCODE) on Aurora B (green) and CMYC (blue) genes showing H2A.Z enrichment at the TSS and at the upstream region, respectively (bottom panels). IGV analyses of ATAC-seq peaks for control si and H2A.Z.1 si for Aurora B and MYC genes. The number in red represents the log2Ratio H2AZ.1 si/Control si.

E Plot representing the average value for all the target regions around the TSS obtained by ATAC-seq after control, H2A.Z.1 or H2A.Z.2 siRNA.

$\mathrm{F}$ Plot representing the number of differential chromatin accessibility regions in pairwise comparison. Green (up) represents regions with increased accessibility and red (down) with decreased accessibility.

G Distribution of the position within the nucleus of the centromere of $\mathrm{Chr} 17$ from the experiment in $(\mathrm{H})$ calculated as the ratio $\mathrm{d} 2 / \mathrm{d} 1$. The ratio between $\mathrm{d} 2$ and $\mathrm{d} 1$ gives the position of the centromere relative to the centre of the nucleus. The graph represents the percentages of centromeres with distances following within the 5 binning categories. At least 500 nuclei were analysed per condition. Data sets were statistically analysed using Chi-square test for the distribution of signals among the 5 categories. ${ }^{* \star *} p<0.001$

$\mathrm{H}$ Representative image of a HeLa nucleus after FISH with Chr17 centromeric probe (green). The distance of the FISH signals from the nucleus periphery was calculated as follows: the distance from the centre of the nucleus to the periphery (d1) and the distance from the centre of the nucleus to the FISH signal ( $\mathrm{d} 2$ ) were measured. (scale bar $5 \mu \mathrm{m}$ ) 
A

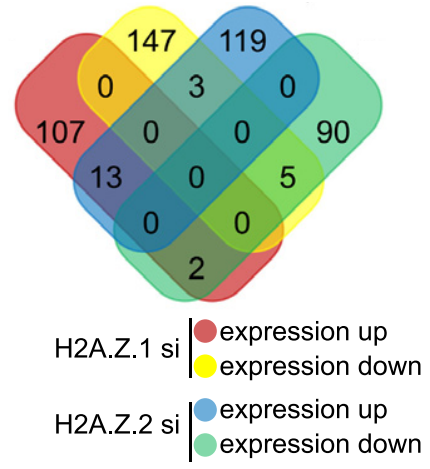

B

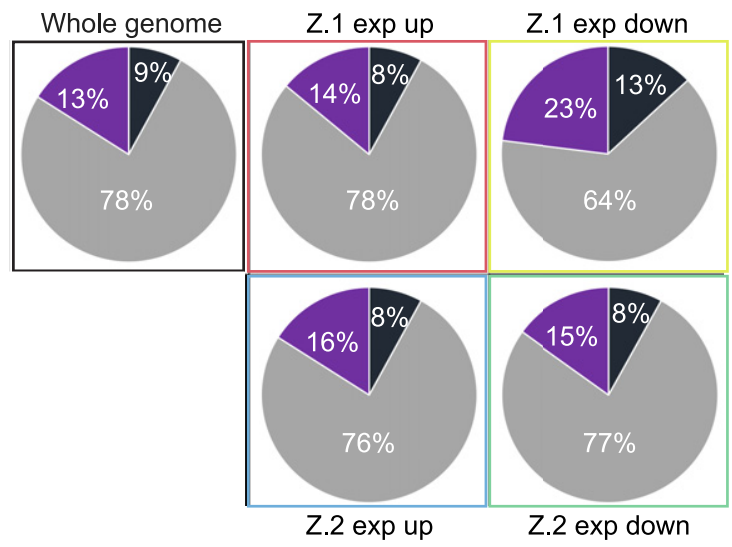

- TSS $\pm 1 \mathrm{~Kb}$

Gene body

Others

Whole genome Z Z.1si- exp up ${ }^{\mathrm{ns}}$ - Z.1si- exp down ${ }^{\text {ns }}$ ns Z.2si- exp up Z.2si- exp down

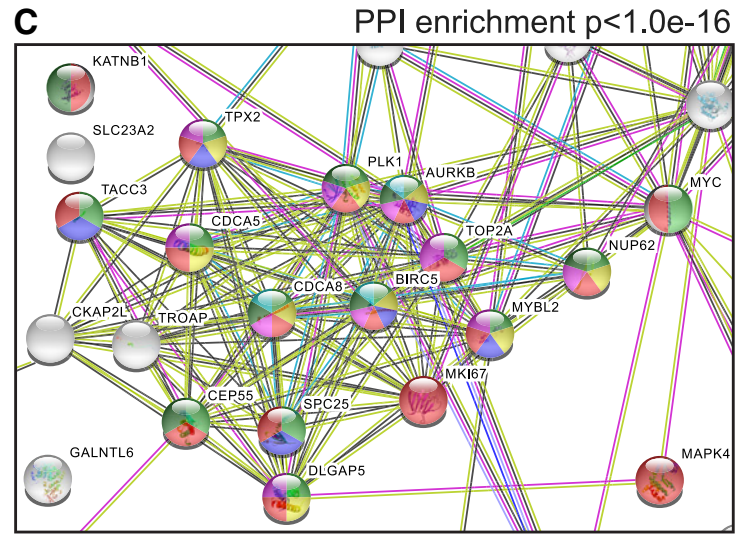

\section{GO terms}

- Microtubule cytoskeleton organization involved in mitosis

Mitotic cell cycle process

Mitotic nuclear division

- Nuclear division

- Cell cycle

CPC

Midbody
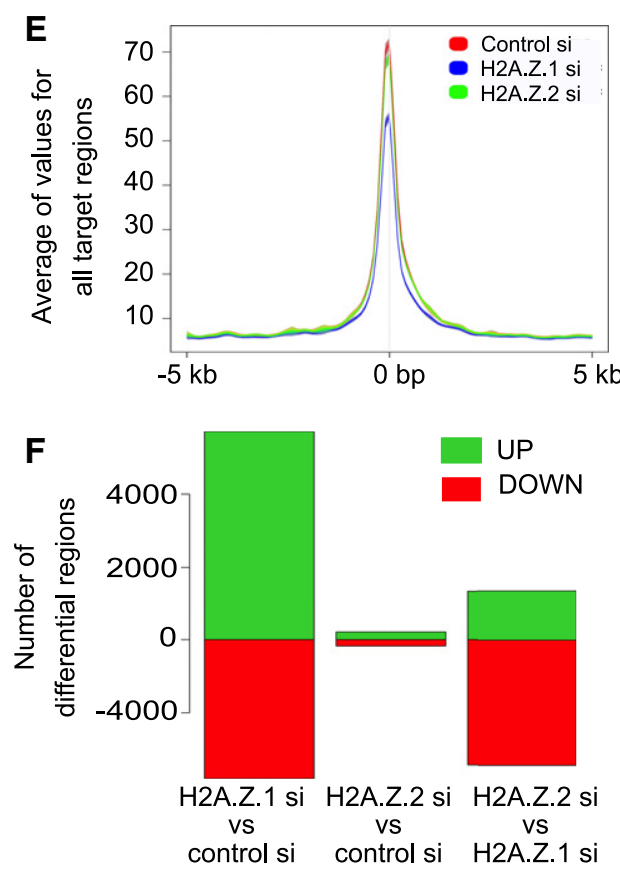

D
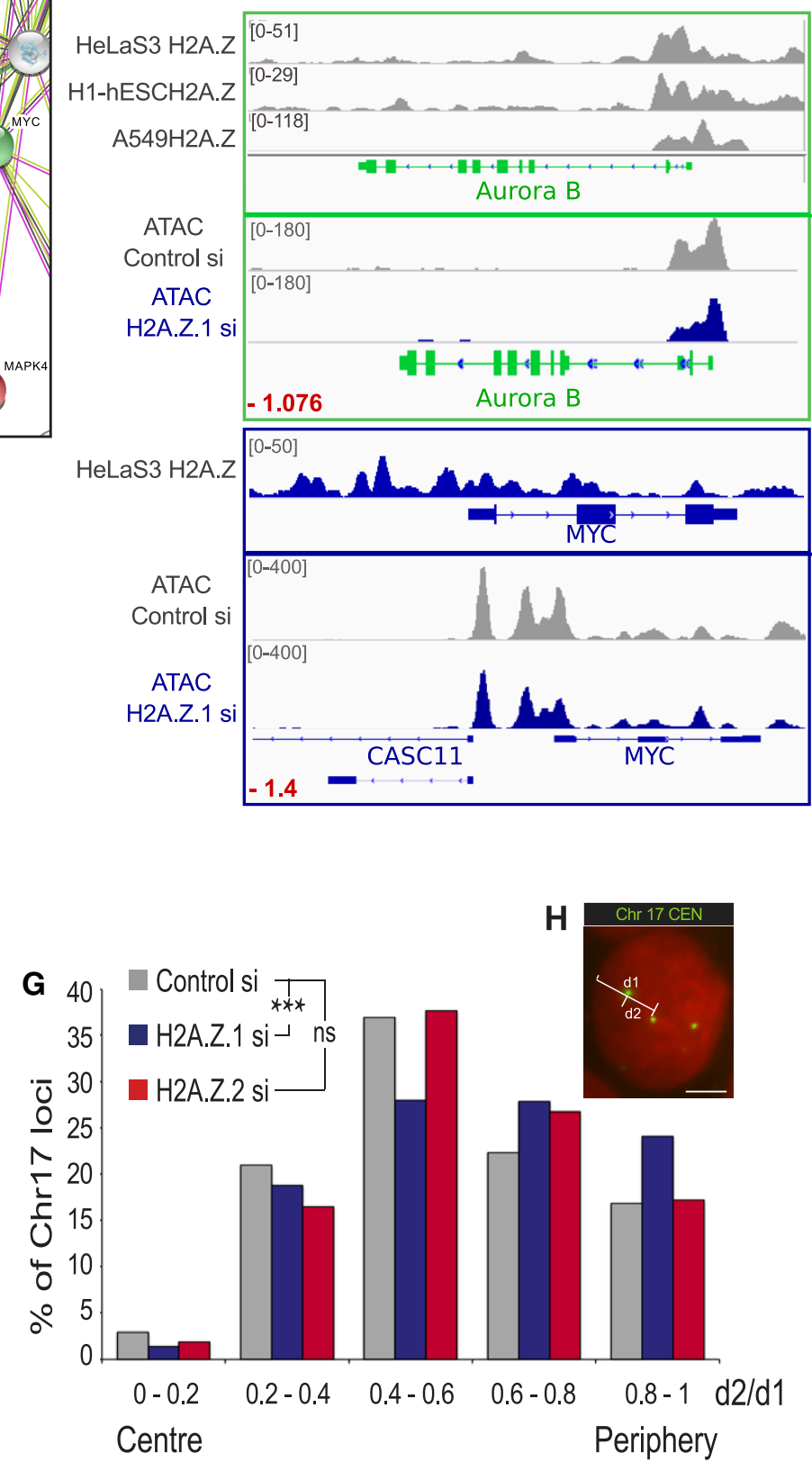

Figure 3. 
decrease in mitotic index (Figs 2G and EV2G). In fact, FACS analyses of H2AZ.1-downregulated cells revealed a significant change in the profile with an increase in $\mathrm{G} 1$ phase and a reduction in $\mathrm{S}$ (Fig 4A), thus suggesting a G1/S arrest. H2A.Z.1-depleted cells do not proceed through the cell cycle. The pathways involved in the G1 checkpoint are well characterised; therefore, we checked whether the list of genes affected by H2A.Z.1 depletion included some of the known regulators. This was indeed the case: both the cyclindependent kinase inhibitor $1 \mathrm{~A}(\mathrm{CDKN} 1 \mathrm{~A})(\mathrm{p} 21)$ and $1 \mathrm{~B}$ (CDKN1B) (p27) were significantly upregulated upon H2A.Z.1 but not H2A.Z.2 RNAi (Fig 4B). Interestingly, both these genes have H2A.Z at their promoters (Fig EV3A). This phenotype was at contrast with the one obtained after H2A.Z.2 depletion where no changes in the cell cycle profile were obtained (Fig EV2F).

To corroborate these findings, we conducted a GSEA analyses of genes that are upregulated and downregulated upon H2A.Z.1 depletion.

These analyses revealed a significant enrichment in genes that are directly bound and regulated by TP53 $(P=3.82 \mathrm{e}-17)$ for the upregulated genes: this again suggests a p53-mediated cell cycle arrest. On the contrary, among the genes that are downregulated, there was a significant enrichment for mitotic and cell cycle genes and target genes of the DREAM repressive complex that promotes cell cycle exit by repressing cell cycle genes (Uxa et al, 2019; $P=2.65 \mathrm{e}-144$ and $2.16 \mathrm{e}-14$, respectively, Fig $4 \mathrm{C}$ ); this further indicates a block in the cell cycle programme upon H2A.Z.1 depletion. As p21 can mediate cellular senescence via p53dependent and p53-independent pathways (Abbas \& Dutta, 2009; Qian \& Chen, 2010), we investigated whether H2A.Z.1-depleted cells were in a senescence status by assessing the expression of senescence-associated $\beta$-galactosidase. We indeed found that $80 \%$ of H2A.Z.1-depleted cells were $\beta$-Gal positive (Fig 4D). As an independent marker for senescence, we also quantified the levels of Ki-67 in H2A.Z.1-depleted cells and found a significant increase in cells with weak or no staining for this proliferation marker (Fig 4E and F).
These transcriptional and cell cycle changes were also associated with the presence of an aberrant nuclear shape (Fig 4G), a defect not evident in H2A.Z.2-depleted cells. To quantify the phenotype, we analysed the nuclear circularity of control, H2A.Z.1- and H2A.Z.2depleted cells using the NIS Elements AR Analysis software (NIKON); the results showed a significant decrease in the circularity of nuclei upon H2A.Z.1 knockdown (Fig 4H). To confirm that the phenotype was specifically due to H2A.Z.1 depletion and to analyse whether its post-translational modifications were necessary for this function, we generated GFP-tagged oligo-resistant constructs for H2A.Z.1 ${ }^{\text {WT }}$ and H2A.Z.1 ${ }^{\mathrm{KR}}$ : both constructs were able to rescue the phenotype (Fig 4I). These data indicate that H2A.Z.1 is required to maintain a round nuclear shape, but its post-translational modifications are dispensable for this function. H2A.Z.2 also alters the roundness of the nuclear shape but this is due to the presence of micronuclei close to the main nucleus rather than by the irregular overall morphology.

\section{H2A.Z.1 regulates cell cycle progression via MYC}

Since we have revealed a decrease in cMYC in the RNA-seq experiments together with a change in the chromatin upstream upon ATAC-seq analyses, we wanted to test if the cell proliferation block could be due to MYC. We therefore expressed MYC in cells depleted of H2A.Z.1 and evaluated the effect on the cell cycle by analysing the mitotic index. As previously mentioned, H2A.Z.1-depleted cells have a major decrease in the mitotic index but MYC overexpression can overcome the cell cycle block and significantly increase cell proliferation (Fig 5A).

Because our data indicate a role of H2A.Z.1 in the maintenance of cell proliferation via MYC regulation, we reasoned that these results could have important implications in cancer. MYC activation is the most frequent molecular alteration observed in human cancers, and among those, neuroblastoma represents a classic example. Amplification of the MYCN transcription factor is a common feature of advanced neuroblastoma and associated with a poor outcome of the disease. We therefore investigated whether a

Figure 4. H2A.Z.1 depletion causes $\mathrm{G} 1$ arrest and cell senescence.

A Flow cytometry analysis profiles of control (top) and H2A.Z.1 (bottom) siRNA-treated HeLa cells. Percentages represent the mean of two biological replicates. Data sets were statistically analysed using Chi-square test. ${ }^{\star \star *} P<0.001$.

B CDKN1A (top) and CDKN1B (bottom) average expression values obtained by RNA sequencing of three biological replicates after control, H2A.Z.1 (Z1) or H2A.Z.2 (Z2) siRNA treatment. Error bars show the standard deviation (SD). *** $P<0.001$ (Student's $t$-test).

C GSEA analyses of the upregulated (light blue upwards arrow) and downregulated (light green downwards arrow) genes upon H2A.Z.1 siRNA indicating the number of genes for category and the relative $P$-values.

D Representative images of HeLa cells transfected with control, H2A.Z.1 or H2A.Z.2 siRNA and stained for senescence-associated $\beta$-galactosidase (blue). Scale bar $200 \mu \mathrm{m}$. At the bottom-left, quantification of the percentage of $\beta$-galactosidase-positive cells. At least 500 cells were counted for each experiment. Error bars show the standard deviation (SD) from two biological replicates. ${ }^{* *} P<0.01 ;{ }^{* \star *} P<0.001$ (Student's $t$-test).

E Representative images of HeLa cell stained for Ki-67. The arrows indicate the different staining patterns quantified: strong signal (grey arrow), weak signal (light brown arrow) and no signal (dark brown arrow; scale bar $10 \mu \mathrm{m}$ ).

F Quantification of the experiment in (E). Error bars indicate the standard deviation from three biological replicates. Data sets were statistically analysed using a Chi-square test. ${ }^{* *} p<0.001$.

G Representative images of HeLa cells transfected with control (top) or H2A.Z.1 (bottom) siRNA and stained for Lamin A/C (green). Scale bar $=10 \mu \mathrm{m}$.

$\mathrm{H}$ Violin plots of the nuclear circularity index of HeLa cells transfected with control (grey), H2A.Z.1 (blue) or H2A.Z.2 (red) siRNA. The nuclear circularity was analysed using the NIS Elements AR Analysis software (NIKON). At least 140 nuclei from three biological replicates were analysed for each condition. Mean and SD are shown. Data sets were statistically analysed using the Wilcoxon rank test in $\mathrm{R}$. ${ }^{* \star *} P<0.001$

I Violin plots of the nuclear circularity index of HeLa cells transfected with control (grey) or H2A.Z.1 (blue) siRNA either alone or in combination with siRNA-resistant GFP:H2A.Z.1 ${ }^{\text {WT }}$ (WT) or GFP:H2A.Z.1 ${ }^{\text {KR }}$ (KR) plasmids. The nuclear circularity was analysed using the NIS Elements AR Analysis software (NIKON). At least 140 nuclei from three biological replicates were analysed for each condition. Mean and SD are shown. Data sets were statistically analysed using the Wilcoxon rank test in R. ${ }^{\star * \star} P<0.001$. 
A

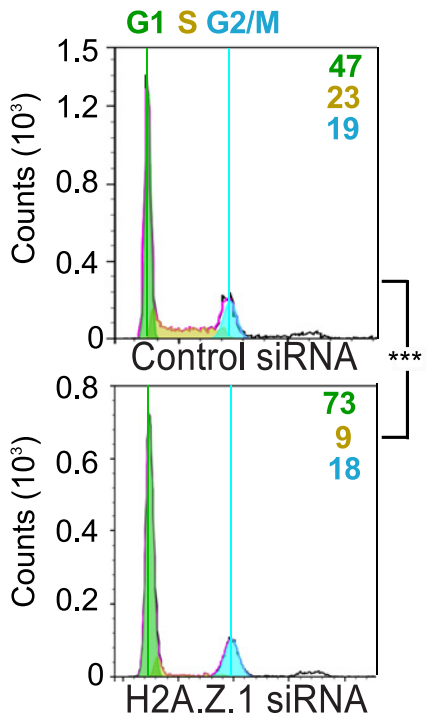

B

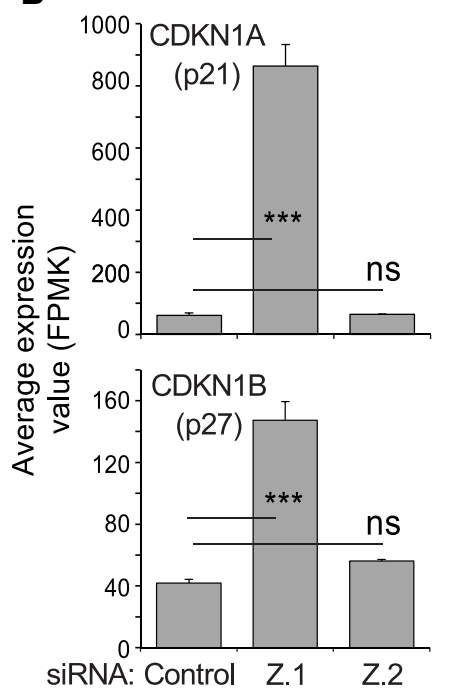

C

\begin{tabular}{|c|c|c|}
\hline Category & Genes & $p$ values \\
\hline $\begin{array}{l}\text { Genes directly bound and regulated } \\
\text { by TP53 GenelD7157 }\end{array}$ & 17 & $3.82 \mathrm{e}-17$ \\
\hline $\begin{array}{l}\text { SENESE_HDAC1_AND_HDAC2 } \\
\text { Targets_DN [238] }\end{array}$ & 11 & $1.06 \mathrm{e}-10$ \\
\hline Target genes of the DREAM complex & 25 & $2.16 \mathrm{e}-14$ \\
\hline Mitotic_Cell cycle & 26 & 2.65 e-14 \\
\hline
\end{tabular}

D

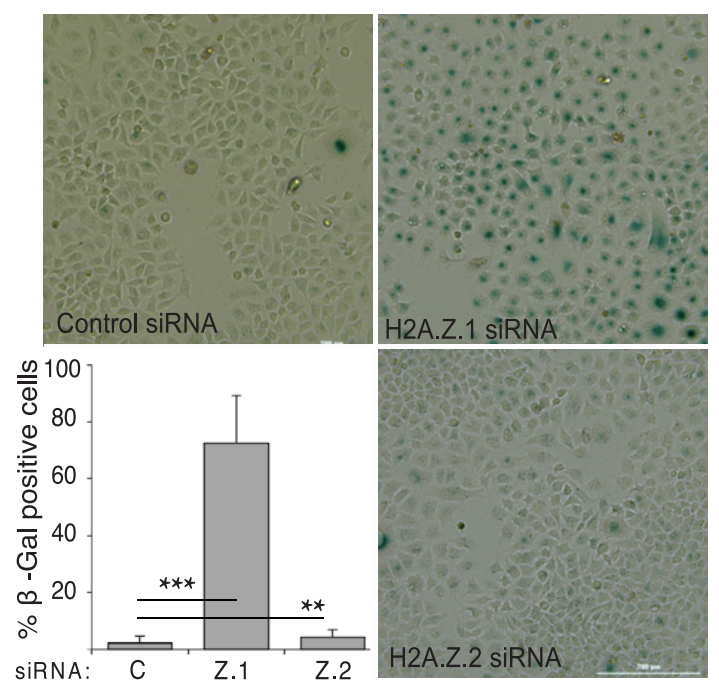

E

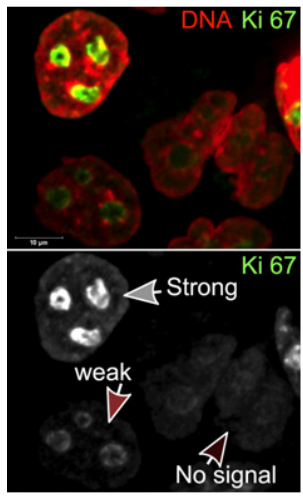

F

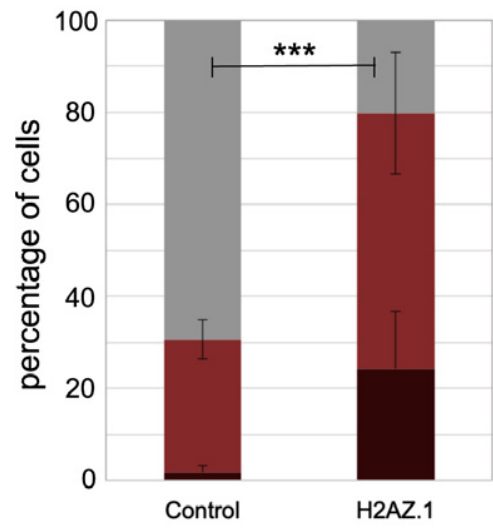

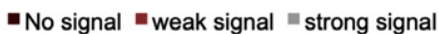

G

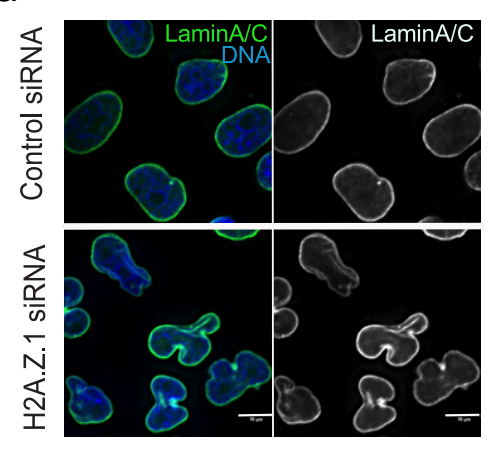

H

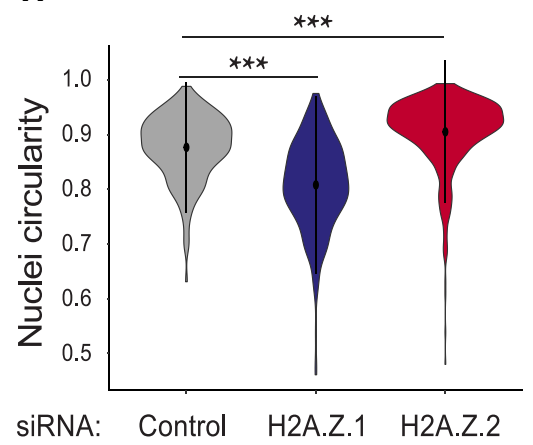

I

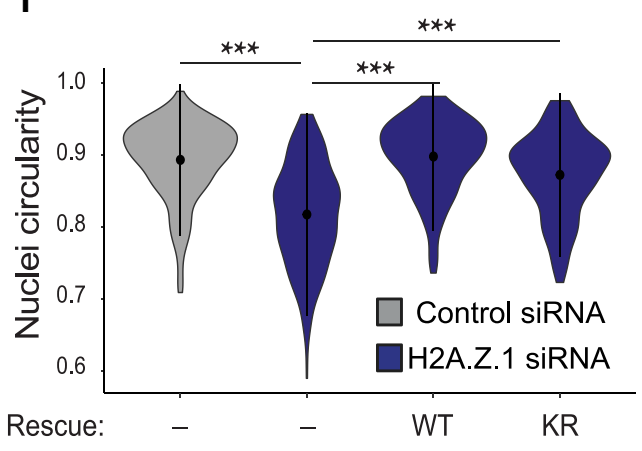

Figure 4. 
A

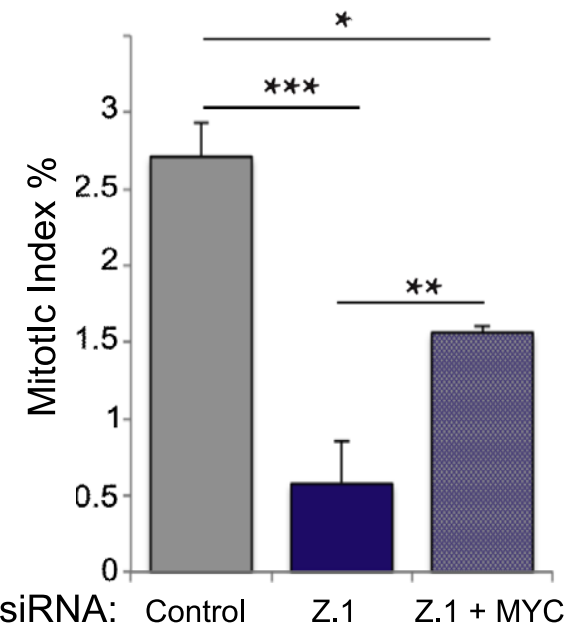

C

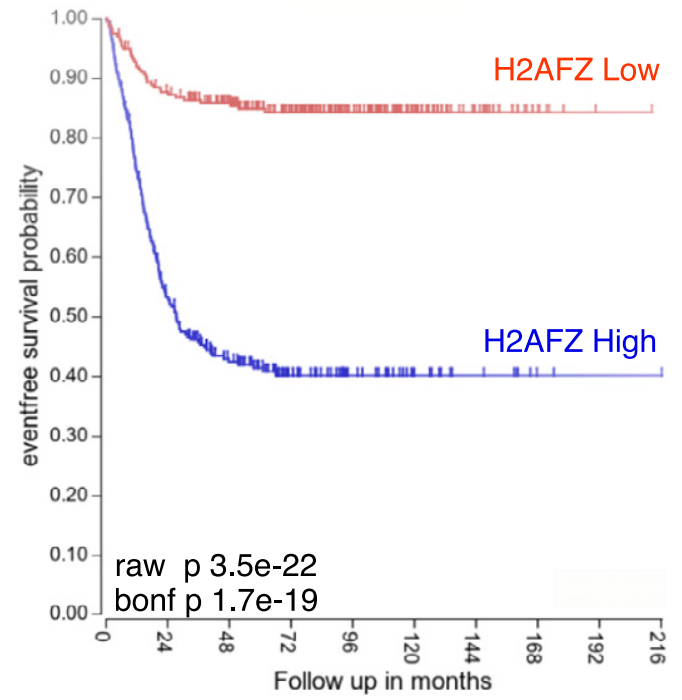

B

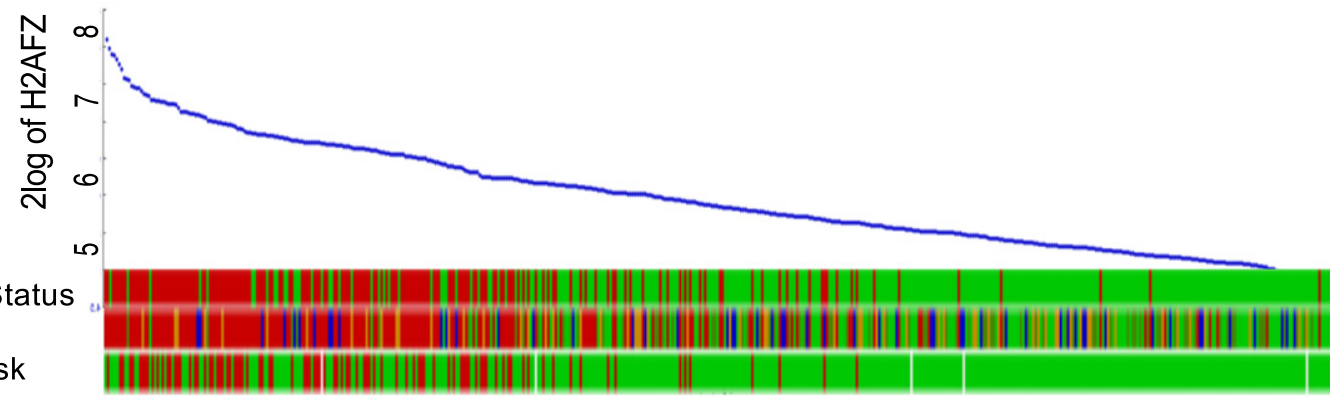

Figure 5. H2A.Z.1 effect on cell cycle is linked to MYC expression.

A Mitotic index (number of mitotic cells/mitotic + interphase cells) of HeLa cells after control si, H2A.Z.1 si or H2A.Z.1 si + GFP:CMYC. Error bars represent standard deviation from two biological replica (control si $N=1,033$; H2A.Z.1 si $N=1,042 ;$ H2A.Z.1 si + GFP:CMYC $N=1,024$ ). Data sets were statistically analysed using Chisquare test. ${ }^{*} P<0.05 ;{ }^{* *} P<0.01 ;{ }^{* \star *} P<0.001$.

B Correlation of H2AFZ (H2A.Z.1) expression levels with MYCN status, cancer stage and risk incidence in neuroblastoma patients obtained from R2 genomics.

C Kaplan-Meier survival curve for neuroblastoma patients with low (red) or high (blue) expression of H2AFZ (H2A.Z.1) obtained from R2 genomics.

correlation between H2A.Z.1 and MYCN exists in neuroblastoma tumours using the R2 genomic platform. We stratified the patients for MYC amplification and stage of the tumour. The results (Fig 5B) clearly show a correlation between H2A.Z.1 level and both MYCN level and clinical stage. Moreover, a Kaplan-Meier analysis also revealed that neuroblastoma patients expressing high level of H2A.Z.1 have a worse prognosis compared with those presenting a low expression level (Fig 5C). This is almost reminiscent of the prognostic value of MYCN itself for this type of cancer.

Therefore, we can conclude that the biological importance of H2A.Z.1 in promoting cell proliferation via favouring Myc transcription and suppression of p21/p27 and could be extremely important in cancer as prognostic indicator but also as possible therapeutical avenue.

In summary, our data suggest that the two H2A.Z paralogues have distinct roles in the regulation of cell cycle progression: while H2A.Z.1 acts at the G1/S transition by suppressing the expression of p21 and p27 thus facilitating the expression of cell proliferation genes, H2A.Z.2, in a transcription-independent manner, contributes to the maintenance of centromeric cohesion and recruitment of spindle assembly checkpoint proteins, thus playing a role at the M/ G1 transition (Fig 6).

\section{Discussion}

Despite the established importance of the histone variant H2A.Z in many aspects of cell biology and growing evidence suggesting differential roles for the H2A.Z.1 and H2A.Z.2 paralogues in chromatin organisation and function, the majority of the studies thus far have focussed on H2A.Z as a single variant and very little is known about their individual roles (if any) during cell cycle in mammals. In this study, we have shown that H2A.Z.1 and H2A.Z.2 have specific and distinct functions in chromosome segregation and cell cycle progression in human cells.

\section{H2A.Z.2 is essential for chromosome segregation}

H2A.Z.2-specific depletion in HeLa cells resulted in profound chromosome segregation errors that led to micronuclei formation. Our 


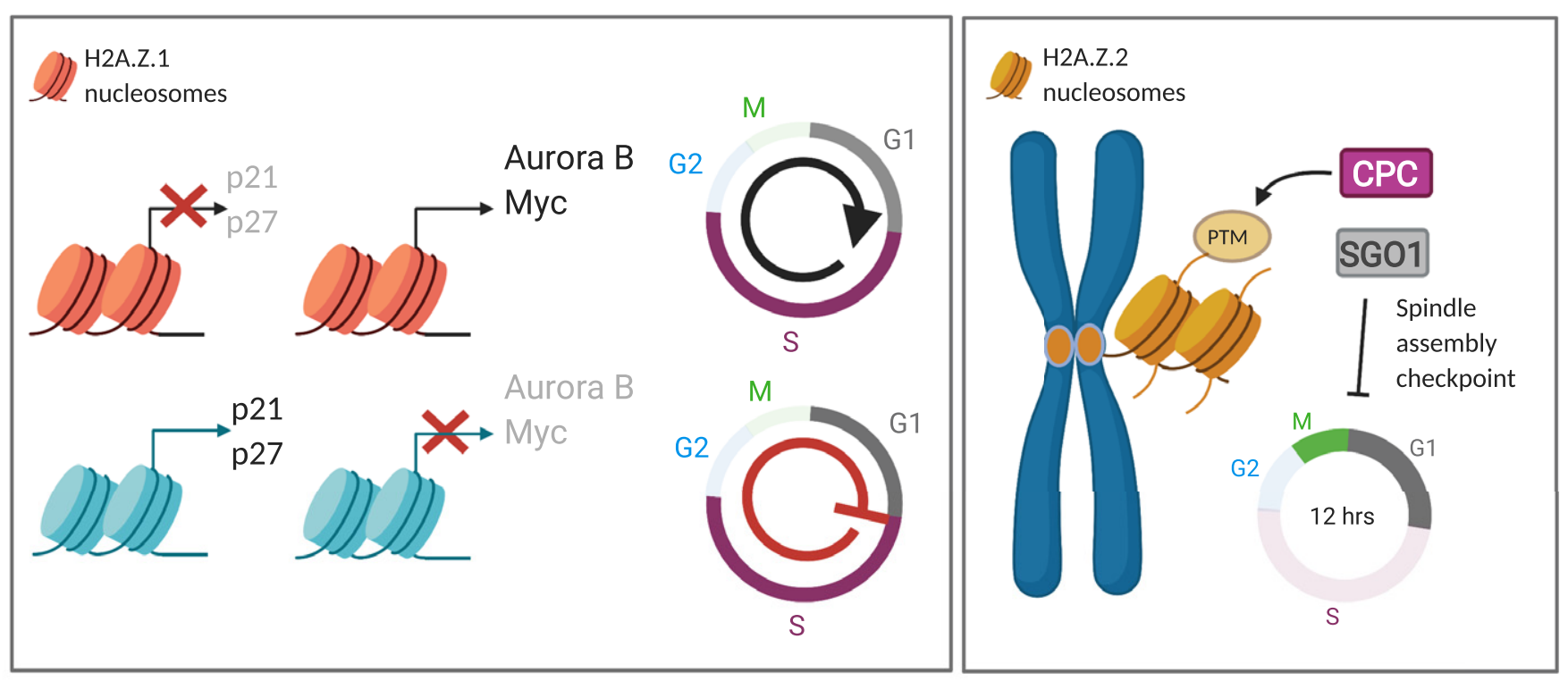

Figure 6. Model for the distinct roles of H2A.Z.1 and H2A.Z.2 in cell cycle regulation.

(Left panel) H2A.Z.1 promotes the expression of several cell cycle genes whilst repressing the G1/M checkpoint proteins p21 and p27, thus favouring cell cycle progression. Upon downregulation of H2A.Z.1, the expression of these genes is altered leading to G1/S cell cycle arrest and cellular senescence. (Right panel) H2A.Z.2, through its post-translational modifications, regulates the recruitment of several centromeric proteins and contributes to the regulation of the spindle assembly checkpoint. H2A.Z.2 depletion leads to a defective SAC, premature sister chromatid separation and chromosome segregation errors.

study has provided clear evidence for the different roles of the splice variants and their post-translational modifications that are essential for chromosome segregation. The isoform H2A.Z.2 is critical for chromosome segregation, and this function depends on its posttranslational modification status (or it is mediated by other changes linked to the residues that can potentially be modified), since we have demonstrated that both the wild-type and mutant forms can be incorporated into the chromatin with the same efficiency, but the mutant cannot rescue the segregation defect phenotype. Although the shorter H2A.Z.2.2 variant possesses the acidic patch responsible for its deposition, it lacks the C-terminal tail, which has been shown to be important in Drosophila (Clarkson et al, 1999) and yeast (Wratting et al, 2012). The C-terminal tail contains the A127 residue that seems to be important for chromosome segregation and several residues that can be post-translational modified and could potentially have an impact either on the stability of the nucleosomes or the recruitment of specific readers. In fact, the mutation of residue 127, although still effective to some extent in ameliorating the micronucleation phenotype, when in combination with either residue 14 or 38 was not. Amino acid 38 is important for the L1 loop structure and nucleosome stability (Horikoshi et al, 2013), and the S38T substitution in H2A.Z.1, mimicking T38 found in H2A.Z.2, can rescue the SRCAP-dependent Floating-Harbor syndrome (Greenberg et al, 2019) defects. The role of the amino acid 14 is still unknown but being an alanine that cannot be phosphorylated (compared to the threonine in the paralogue counterpart), it could suggest that a differential phosphorylation between the two forms may play a critical role, especially during mitosis.

We have investigated the molecular mechanisms behind the chromosome segregation defects. H2A.Z.2-depleted cells presented impaired loading of CENP-A, CENP-C, Sgo1 (protector of centromeric cohesion) and Aurora $\mathrm{B}$ (member of the $\mathrm{CPC}$ ). Interestingly, two H2A.Z-specific peptides (GDEELDSLIK and ATIAGGGVIPHnote that these sequences are present in both H2A.Z.1 and H2A.Z.2) were found enriched in the CENP-A nucleosome pull-downs when compared with those containing histone H3.1 (Foltz et al, 2006). This suggests that a specific centromeric CENP-A containing chromatin could favour the maintenance or deposition of CENP-A. Interestingly, the majority of H2A.Z deposition at pericentric heterochromatin occurs in G1 (Boyarchuk et al, 2014), at the same time as CENP-A. Treatment with 5'-aza 2'-deoxycytidine (5-Aza) leads to an increased incorporation of both CENP-A and H2A.Z at pericentric heterochromatin that has been interpreted as a consequence of a disruption in the heterochromatin at the centromere (Boyarchuk et al, 2014). However, we do not see a loss of HP1 $\alpha$ at the centromere; therefore, our data seem to suggest that H2A.Z.2 incorporation is upstream and can affect CENP-A deposition or stability. This is a novel aspect that will be further investigated.

We have also observed a decrease in Aurora B levels at centromeres of mitotic chromosomes in cells depleted of H2A.Z.2. It was previously reported that INCENP ${ }^{360-876}$ could interact in vitro with H2A.Z and that the GDEELDSLIKA region was essential for the binding (Rangasamy et al, 2003). This could explain the results we have obtained, but it does not account for the specificity; in fact, the interacting region is the same in both paralogues. Our results reveal for the first time a link between H2A.Z.2 and sister chromatid cohesion. The known pathways connecting the CPC and Sgol loading are mediated by HP1 $\alpha$ (Yamagishi et al, 2010; Ruppert et al, 2018; Nonaka et al, 2002; Inoue et al, 2008; Perera \& Taylor, 2010; Kang et al, 2011). However, H2A.Z.2 depletion does not seem to alter 
HP1 $\alpha$ pericentromeric localisation, thus indicating that we have uncovered a possible novel pathway important for the maintenance of these centromeric proteins in mitosis. The CPC is also maintained at the centromere via the phosphorylation of H3T3 (Kelly et al, 2010). Interestingly, depletion of H2A.Z.2 leads to an abnormal distribution of this phosphorylation which spreads into the chromosome arms, while it is normally only localised at the centromeric chromatin. This could contribute to the impaired localisation of the CPC. The link between H2A.Z and the centromeric chromatin structure is not known and could be an important avenue to investigate.

Finally, the data we have provided on the correlation between CENP-A and the Sgo1 levels at centromere suggest that H2A.Z.2 is a key chromatin player at the intersect of different pathways that altogether contribute to maintain a functional centromere.

\section{H2A.Z.1 and H2A.Z.2 have separate functions in gene expression regulation}

The RNA-seq analyses also revealed non-overlapping roles for H2A.Z.1 and H2A.Z.2 in transcription regulation. These distinct effects could be due to the different binding partners reported for H2A.Z.1 and H2A.Z.2: H2A.Z.1 has preference for interacting with BRD2, PHF4, HMG20A and TCF20, whereas H2A.Z.2 has been shown to interact with SIRT1 (Draker et al, 2012; Punzeler et al, 2017; Lamaa et al, 2020). A recent study conducted in U2OS and fibroblast cells analysed the target transcriptomic landscape after individual depletion of each H2A.Z variant; the results showed both distinct and overlapping sets of genes, as well as similar or antagonistic functions, depending on the targets (Lamaa et al, 2020). Our data sets differ from the reported one as we obtained an almost nonoverlapping picture of gene expression changes and we observed a significant enrichment of H2A.Z at the TSS of genes that undergo downregulation after H2A.Z.1 depletion. In agreement with this study, our data have shown that H2A.Z.1 depletion resulted in downregulation of a subset of cell cycle-regulated genes, which explains the cell cycle arrest and low cell division rate we have reported here. However, we did not found downregulation of cell cycle-related genes after H2A.Z.2 depletion. The ATAC-seq analyses also revealed distinct effects on chromatin reorganisation upon H2A.Z.1 or H2A.Z.2 RNAi with H2A.Z.1 having a more prominent impact on chromatin than H2A.Z.2.

We have also shown that the cell cycle mis-regulated genes upon H2A.Z.1 depletion do contain H2A.Z at their promoters, suggesting a direct effect played by the histone variant rather than being a secondary effect; only a few showed changes upon ATAC-seq analyses. Among those genes, we have identified MYC as one of the key players that mediate H2A.Z.1 effect. In fact, p21, a classic regulator of the G1/S checkpoint, has been reported to be one of the major targets of MYC repression activity (Coller et al, 2000). Upon H2A.Z.1 knockdown, MYC expression levels decrease and the ATAC peaks upstream the gene show also a 1.4-fold decrease; this leads to increased p21 mRNA, activation of the G1/S checkpoint that results in decreased proliferation (low mitotic index), G1 arrest and a senescent phenotype. This effect can be overridden by ectopically expressing cMYC. These data seem to suggest a direct link between MYC and H2A.Z.1

The senescent phenotype is associated with an abnormal nuclear morphology which can be reminiscent of the p53-mediated cellular senescence pathway mediated by lamin A stabilisation (Yoon et al, 2019). However, we did not detect any significant change in lamin A expression level upon H2A.Z.1 RNAi.

Based on the data we have presented on the effect of these paralogues in cell cycle progression and the observed overexpression of H2A.Z in a variety of malignant tumours, including breast (Hua et al, 2008), prostate (Slupianek et al, 2010), bladder (Kim et al, 2013b) cancers and metastatic melanoma (Vardabasso et al, 2015), we should consider the relevance of this histone variant in cancer. Here, we have also provided data linking high expression of H2A.Z.1 to MYCN as bad prognostic feature in Neuroblastoma. Moreover, the co-regulation of MYC, the $\mathrm{CPC}$ and its counteracting phosphatase Repo-Man (CDCA2) by H2A.Z.1 we have here reported, provides further support to the important role of these complexes in cancer where the co-upregulation of Repo-Man and Aurora B in tumours is inversely correlated with patient survival (Manzione et al, 2020). This again underlines the potential importance of H2A.Z.1 for cancer progression, acting as an oncogene.

Some studies suggested the possibility that it is the balance between the two paralogues that allows for a normal cell homeostasis. In this respect, since we have shown that H2A.Z.2 depletion results in chromosome instability while H2A.Z.1 supports the expression of master cell cycle genes, we can envisage that slight changes in their balance could easily produce an aberrant cancer prone phenotype, on the one hand by supporting proliferation (High H2A.Z.1) and on the other hand by increasing genome instability (Low H2A.Z.2), both beneficial for a tumour cell to evolve. In this respect, it would be interesting to analyse CIN tumours and correlate their evolution with the expression level of H2A.Z.2.

In conclusion, we have demonstrated non-redundant roles for H2A.Z.1 and H2A.Z.2 in different aspects of cell biology and gene expression, highlighting the importance of studying these variants independently. More studies will be required to unveil the details of the downstream effectors of these paralogues, the chaperones and machineries dedicated to the specific deposition of these variants. This is particularly important for therapeutic purposes, as only targeting the correct variant would offer a proper intervention.

\section{Materials and Methods}

\section{Cell culture, cloning and transfections}

HeLa cells were grown in DMEM supplemented with $10 \%$ foetal bovine serum (FBS) and $1 \%$ penicillin-streptomycin (Invitrogen Gibco) at $37^{\circ} \mathrm{C}$ with $5 \% \mathrm{CO}_{2}$.

DT40 cells carrying a single integration of the LacO array (de Castro et al, 2017) were cultured in RPMI1640 supplemented with $10 \%$ FBS, $1 \%$ chicken serum and $1 \%$ penicillin-streptomycin at $39^{\circ} \mathrm{C}$ and $5 \% \mathrm{CO}_{2}$.

The HeLa CENP-A:YFP cell line was kindly provided by Dr Lars E.T. Jansen (Oxford University, UK).

Transient transfections for DT40 in LacO array background were conducted as previously described using GFP-fused H2A.Z.1, H2A.Z.2.1 and H2A.Z.2.2 (Vagnarelli et al, 2011).

For siRNA treatments, HeLa cells were seeded into 6-well plates, transfected using Polyplus JetPrime ${ }^{\circledR}$ (PEQLAB, Southampton, UK) with the appropriate siRNA oligonucleotides $(50 \mathrm{nM})$ and analysed 
after $72 \mathrm{~h}$. The siRNAs were obtained from Merck. Control: $5^{\prime}$-CG UACGCGGAAUACUUCGA-3'; H2A.Z.1: 5'-GCCGUAUUCAUCGACA CCU-3'; H2A.Z.2\#1: 5'-AUUUGUAUGUUCUUAGACU-3'; H2A.Z.2\#2: $5^{\prime}$-GUGACAGUUGUGUGUUGAU- ${ }^{\prime}$.

For rescue experiments, $1 \mu \mathrm{g}$ of GFP:H2A.Z.1 DNA or $100 \mathrm{ng}$ of GFP:H2A.Z.2 DNA was used. GFP:H2A.Z.1 ${ }^{\text {WT }}$, GFP:H2A.Z.2.1 ${ }^{\text {WT }}$, GFP:H2A.Z.2.2 ${ }^{\text {WT }}$, GFP:H2A.Z.1 ${ }^{\mathrm{KR}}$, GFP:H2A.Z.2.1 ${ }^{\mathrm{KR}}$ and GFP: H2A.Z.2.2 ${ }^{\mathrm{KR}}$ were synthetised by ProteoGenix (La Haye, France). They were all cloned into pEGFP-C1 by Xhol/ KpnI, except GFP: H2A.Z.2.1 ${ }^{\text {WT }}$ and GFP:H2A.Z.2.2 ${ }^{\text {WT }}$ that were cloned by BglII/ BamHI1 and BglII/ EcoRI, respectively. Single or double mutations were produced with the $\mathrm{Q} 5^{\circledR}$ Site-directed Mutagenesis kit (New England Biolabs, UK) using the primers in Table 1.

The oligo-resistant H2A.Z.1 mutants were generated by mutating the following oligo target sequence GGC(G) CGT(R) ATT(I) CAT(H) CGA(R) CAC(H) CTA(L) to GGC(G) $\underline{A G G(R) ~ A T \underline{C}(I) ~ C A T(H) ~} \underline{A G G}$ (R) $\mathrm{CAC}(\mathrm{H}) \mathrm{CTA}(\mathrm{L})$.

To produce the RFP:LacI:YL1 construct, we first generated a GFP:LacI:YL1 plasmid. The YL1 sequence was obtained by PCR using the primers in Table 1 and cloned into GFP:LacI (de Castro et al, 2017) by HindIII/BamHI. GFP was replaced by RFP using NheI/BglII.

GPS-MYC was a gift from Channing Der (Addgene plasmid \# 160130; http://n2t.net/addgene:160130; RRID:Addgene_160130) (Blake et al, 2019).

The primers used for the study were acquired from Eurofins Genomics (Germany) and all the restriction enzymes from New England Biolabs (UK).

Table 1. List of primer used in the study

\begin{tabular}{|c|c|c|}
\hline Name & Primer & Sequence ( $5^{\prime}$ to $\left.3^{\prime}\right)$ \\
\hline \multirow[t]{2}{*}{ H2A.Z.2.1A14T } & Forward & GAAGGCCAAGACAAAGGCAGTATC \\
\hline & Reverse & ССАСТGTCСTTTCCAGCT \\
\hline \multirow[t]{2}{*}{ H2A.Z.2.1T38S } & Forward & ACACTTCAACtctCGCACCACAAGC \\
\hline & Reverse & CTGTGGATGCGGCCCACA \\
\hline \multirow[t]{2}{*}{ H2A.Z.2.1A127V } & Forward & GCACAAAACTgtCTAGGGATCCAC \\
\hline & Reverse & TGTCCCTTCTTTCСAATC \\
\hline \multirow[t]{2}{*}{ YL1 } & Forward & AAGCTTTTATGAGTTTGGCTGGGGGCC \\
\hline & Reverse & GGATCCTCATTTAATGACAATTTTCTCGCGC \\
\hline
\end{tabular}

Table 2. List of antibodies used in the study

\begin{tabular}{llll} 
Antibody & Source & $\begin{array}{l}\text { Cat } \\
\text { number }\end{array}$ & Dilution \\
\hline $\begin{array}{l}\text { Lamin A+Lamin } \\
\text { C }\end{array}$ & Abcam & \#ab108595 & 1:2,500 \\
\hline Alpha-Tubulin & Sigma & \#T5168 & 1:1,000 \\
\hline Shugoshin & Abcam & \#ab58023 & $1: 200$ \\
\hline Aurora B/ AIM1 & Cell Signalling & \#3094 & 1:200 \\
& Technology & & \\
\hline CENP-C & Abcam & \#ab193666 & $1: 200$ \\
\hline Ki-67 & BD Biosciences & \#610968 & $1: 100$ \\
\hline H3T3ph & Abcam & \#ab17352 & $1: 2,000$ \\
\hline
\end{tabular}

\section{Immunofluorescence microscopy}

Cells were fixed in $4 \%$ PFA and processed as previously described (Vagnarelli et al, 2006). Primary and secondary antibodies were used as in Table 2. Fluorescence-labelled secondary antibodies were applied at 1:200 (Jackson ImmunoResearch). Three-dimensional data sets were acquired using a wide-field microscope (NIKON Ti-E super research Live Cell imaging system) with a numerical aperture (NA) 1.45 Plan Apochromat lens. The data sets were deconvolved with NIS Elements AR analysis software (NIKON). Threedimensional data sets were converted to maximum projection using the NIS software, exported as TIFF files and imported into Adobe or Inkscape for final presentation.

For the analyses of the mitotic index, cells stained with DAPI and alpha tubulin were used. At least 500 cells per experimental condition for each biological replica were analysed. Chromosome condensation and spindle morphology were used to categorise the different mitotic stages. For analyses of the spindle assembly checkpoint, HeLa cells were transfected with control, H2A.Z.1 or H2A.Z.2 siRNA for $72 \mathrm{~h}$ and treated with nocodazole $(200 \mathrm{ng} / \mathrm{ml})$ for $0,2,4$ and $6 \mathrm{~h}$.

For quantification of the staining, masks were created around the DAPI nuclei or chromosomes using NIS Elements AR Analysis software. Mean intensity of antibodies signals was extracted and exported to Excel (MS office), and background was subtracted.

Quantification of the kinetochore/centromere staining. (CENP-A, CENP-C) 3D stack images were exported and analysed with the Foci quantification plug-in using Fiji (1_color_auto.ijm) (LedesmaFernandez \& Thorpe, 2015). Background was subtracted, and the mean intensity was used to generate the violin plots.

For quantification of enrichment at the LacO locus, four identical circles were designed: one around the LacI spot, two within the nucleus and one outside the cell. Signal intensities were extracted and the mean of the two circles within the nucleus calculated. The outside circle was used as background and subtracted from both the mean nuclear and the LacI spot, and then, the signal intensity from the LacI was normalised relative to the intensity of the nuclear signal.

Violin plots were generated using the ggplot2 package in R.

\section{Senescence-associated $\beta$-galactosidase staining}

To analyse senescence, HeLa cells were treated with control, H2A.Z.1 or H2A.Z. 2 siRNA for $72 \mathrm{~h}$ and stained for $\beta$-galactosidase using the senescence $\beta$-galactosidase staining kit (Cell Signalling Technology \#9860) following manufacturer's protocol. Cells were imaged with a BioTek Cytation 5 imaging reader.

\section{Immunoblotting}

Whole cell extracts were prepared by direct lysis in $1 \times$ Laemmli sample buffer, separated in SDS-PAGE and transferred onto nitrocellulose membranes. Membranes were blocked with $5 \%$ milk in TBS, and primary antibodies were used as following: anti-GFP (Roche, Cat\#11814460001) anti-H2A.Z (Cell Signalling Technologies, Cat \#2781); anti-HP1 $\alpha$ (Millipore, Cat \#05-689) at $2 \mu \mathrm{g} / \mathrm{ml}$; anti-Aurora B/AIM1(Cell Signalling Technologies, Cat\#3094) at 1:1,000; anti-Shugoshin (Abcam, Cat \#ab58023) at 1:200; anti-tubulin 
(Sigma, Cat\#T5168); anti-GAPDH (ProteinTech, Cat\#10494-1-AP) at $1: 10,000$. HRP secondary antibodies were used at 1:10,000 and LiCor secondary antibodies (LiCor IRDye 800CW and 680RD) at 1:5,000. Membranes were visualised using either the Bio-Rad ChemiDoc XRS system or the LiCor Odyssey system.

\section{FISH}

FISH was performed in HeLa cells as previously described (de Castro et al, 2017) using a probe against chromosome 17 (Mejia et al, 2002).

For the centromere position analyses, a single plane containing each spot was selected. The distance of the spots from the periphery was measured and represented as a fraction of the radius on which each spot belongs.

\section{Flow cytometry cell cycle analysis}

Cells were trypsinised, resuspended and incubated at room temperature for $30 \mathrm{~min}$ in $70 \%$ ice-cold ethanol. Cells were centrifuged at $1,000 \mathrm{~g}$ for $4 \mathrm{~min}$, washed with PBS and the supernatant discarded. The pellet was resuspended in $200 \mu \mathrm{l}$ of RNase A/PBS $(100 \mu \mathrm{g} / \mathrm{ml})$ and incubated for $2 \mathrm{~h}$ at $37^{\circ} \mathrm{C}$ in the dark. Propidium iodide (Fisher Scientific, P3566) was added at a final concentration of $5 \mu \mathrm{g} / \mathrm{ml}$ just before analysing the samples by flow cytometry using the ACEA Novocyte Flow Cytometer. The analysis was performed using the NovoExpress ${ }^{\circledR}$ software.

\section{Micrococcal nuclease treatment}

HeLa cells were transfected with $1 \mu \mathrm{g}$ of GFP, GFP:H2A.Z.2.1 ${ }^{\text {WT }}$ or H2A.Z.2.1 $1^{\mathrm{KR}}$ for $24 \mathrm{~h}$. Cells were lysed using lysis buffer (1 M Tris$\mathrm{HCl}, 2.5 \mathrm{M} \mathrm{NaCl}, 10 \% \mathrm{NP}-40$ ), and the chromatin was extracted by centrifugation at $1,000 \mathrm{~g}$ for $3 \mathrm{~min}$. Chromatin was flash-frozen in liquid nitrogen until ready to use. Chromatin was digested with Micrococcal Nuclease (NEB, $\left.37^{\circ} \mathrm{C}, 30 \mathrm{~min}\right)$ in digestion buffer $(1 \mathrm{M}$ Tris-HC, $1 \mathrm{M} \mathrm{CaCl}_{2}, 0.5 \mathrm{M} \mathrm{MgCl}_{2}, 2 \mathrm{M}$ sucrose).

Digested chromatin was resuspended in Laemmli sample buffer and run in a $10 \%$ acrylamide gel. Anti-GFP antibody (Roche, Cat\#11814460001) was used to detect the construct and the anti-H3 C-terminus (Active Motif, Cat\#39052) as a control.

To verify that chromatin was digested, $1 \%$ of SDS (final concentration) was added to $50 \mu \mathrm{l}$ of digested and undigested chromatin. DNA was extracted with phenol chloroform, precipitated with ethanol and analysed in a $1 \%$ agarose gel.

\section{RNA sequencing}

RNA was collected from siRNA-treated HeLa cells and extracted using the RNeasy PowerLyzer Tissue \& Cells Kit (Qiagen) according to manufacturer's protocol. RNA samples were sent to the Wellcome Trust Genomic Centre (Oxford University, Oxford UK) for wholegenome sequencing, using the Illumina HiSeq4000.

The RNA-seq data were analysed using open-source software from the Tuxedo suite, including TopHat2 (Kim et al, 2013a) and Cufflinks (Trapnell et al, 2010). The paired-end raw reads were mapped to the human reference genome GRCh38 using the annotations from GENCODE 28 (Harrow et al, 2012), with TopHat2 v2.1.1 (Bowtie 2 v2.2.6) under standard conditions. All the sequence and annotation files were downloaded from the Illumina website. The resulting alignments were filtered for high quality hits using SAMtools v0.1.19 (Li et al, 2009) with a minimum selection threshold score of 30. Cufflinks v2.2.1 was used to assemble the mapped reads into transcripts and quantify their expression levels. Finally, Cuffdiff, part of the Cufflinks package, was used to identify differentially transcribed genes between samples. Functional enrichment was analysed using String (stringdb.org), while Venn diagrams were performed in the open software FunRich. Volcano plots were performed using the ggplot package in $\mathrm{R}$ v3.5.0.

The file containing the H2A.Z ChIP-seq data used (E117H2A.Z.narrowPeak.gz. https://egg2.wustl.edu/roadmap/data/byFile Type/peaks/consolidated/narrowPeak/) was filtered using a qvalue threshold of 0.01 . The transcription start sites (TSS) are defined by Abugessaisa et al (2019).

If two or more H2A.Z regions overlap, they were considered as one region. For each set of genes, the H2A.Z regions that were not within $\pm 3 \mathrm{~kb}$ of any of the genes in that set were excluded, and the remainder were classified into 3 categories: regions that overlap and/or are within $\pm 3 \mathrm{~kb}$ of one or more of the TSS associated with a gene whose expression changes after RNAi knockdown; regions that overlap the loci of genes whose expression changes after RNAi knockdown but not any of the TSS associated with those genes; and regions that do not overlap the TSS nor the loci of genes whose expression changed after RNAi knockdown. The number of H2A.Z regions in each category is divided by the total number of regions for that gene set to give the proportion of $\mathrm{H} 2 \mathrm{~A} . \mathrm{Z}$ regions in each category.

IGV was used for the graphic representation of the ChIP-seq data on the gene on the gene of interest.

\section{Differentially expressed gene frequency analyses}

To calculate the expected and observed frequency of differentially expressed genes on each chromosome, we used the gene numbers present on each human chromosome and used the chromosome copy number in HeLa cells integrating both the published data sets (Naumova et al, 2013) and our own experimental data set for chromosomes 17 (this study) and chromosomes 13 and 14 (de Castro et al, 2017).

\section{ATAC-seq}

After 72 h treatment with either control, H2A.Z.1 or H2A.Z.2 siRNA, HeLa cells were harvested and frozen in culture media containing FBS and 5\% DMSO. Cryopreserved cells were sent to Active Motif to perform the ATAC-seq assay. The cells were then thawed in a $37^{\circ} \mathrm{C}$ water bath, pelleted, washed with cold PBS and tagmented as previously described (Buenrostro et al, 2013), with some modifications based on (Corces et al, 2017). Briefly, cell pellets were resuspended in lysis buffer, pelleted and tagmented using the enzyme and buffer provided in the Nextera Library Prep Kit (Illumina). Tagmented DNA was then purified using the MinElute PCR purification kit (Qiagen), amplified with 10 cycles of PCR and purified using Agencourt AMPure SPRI beads (Beckman Coulter). Resulting material was quantified using the KAPA Library 
Quantification Kit for Illumina platforms (KAPA Biosystems) and sequenced with PE42 sequencing on the NextSeq 500 sequencer (Illumina).

Reads were aligned using the BWA algorithm (mem mode; default settings) (Li \& Durbin, 2009). Duplicate reads were removed; only reads mapping as matched pairs and only uniquely mapped reads (mapping quality $\geq 1$ ) were used for further analysis. Alignments were extended in silico at their 3'-ends to a length of $200 \mathrm{bp}$ and assigned to 32-nt bins along the genome. The resulting histograms (genomic "signal maps") were stored in bigWig files. Peaks were identified using the MACS 2.1.0 algorithm at a cut-off of $P$-value 1e-7, without control file and with the -nomodel option. Peaks that were on the ENCODE blacklist of known false ChIP-Seq peaks were removed. Signal maps and peak locations were used as input data to Active Motifs proprietary analysis program, which creates Excel tables containing detailed information on sample comparison, peak metrics, peak locations and gene annotations. For differential analysis, reads were counted in all merged peak regions (using Subread), and the replicates for each condition were compared using DESeq2 (Love et al, 2014).

The analyses of Neuroblastoma data sets were conducted using the R2: Genomics Analysis and Visualisation Platform (http://r2.a mc.nl).

\section{Statistical analyses}

Statistical analyses were performed either in Excel (Chi-square test) or in $\mathrm{R}$ (using the Wilcoxon rank test function, differential expression, lowest smoothing).

\section{Data availability}

All unique/stable reagents generated in this study are available from the Lead Contact with a completed Materials Transfer Agreement.

The RNA-seq data sets and ATAC-seq generated during this study are available at NCBI http://www.ncbi.nlm.nih.gov/bioproject/ 629054 and https://www.ncbi.nlm.nih.gov/geo/query/acc.cgi?acc $=$ GSE173113.

The microscopy images supporting the current study are available from the corresponding author on request and they will be shared via FIGSHARE.

Expanded View for this article is available online.

\section{Acknowledgements}

We thank Prof Sala's group (Brunel University London) and Prof Wendy Bickmore (Edinburgh) for advice and useful discussions. The Vagnarelli lab is supported by the Wellcome Trust Investigator award 210742/Z/18/Z to Paola Vagnarelli. The study on MYC was supported by Kidscan PhD studentship awarded to PV. RSG was a recipient of the Isambard PhD scholarship (2016-2019).

\section{Author contributions}

PV and RSC conceptualised the study; IJDC contributed to methodology; RSG, IJDC, HAA, CS, DCK and PV investigated the study; PV and RSC wrote original draft; all the authors wrote review \& editing; PV involved in funding acquisition; PV, CS and WV supervised the study.

\section{Conflict of interest}

The authors declare that they have no conflict of interest.

\section{References}

Abbas T, Dutta A (2009) p21 in cancer: intricate networks and multiple activities. Nat Reu Cancer 9: 400-414

Abe Y, Sako K, Takagaki K, Hirayama Y, Uchida KS, Herman JA, DeLuca JG, Hirota T (2016) HP1-assisted aurora B kinase activity prevents chromosome segregation errors. Deu Cell 36: 487-497

Abugessaisa I, Noguchi S, Hasegawa A, Kondo A, Kawaji H, Carninci P, Kasukawa T (2019) refTSS: a reference data set for human and mouse transcription start sites. J Mol Biol 431: 2407-2422

Bagchi DN, Battenhouse AM, Park D, Iyer VR (2020) The histone variant H2A.Z in yeast is almost exclusively incorporated into the +1 nucleosome in the direction of transcription. Nucleic Acids Res 48: 157-170

Bargaje R, Alam MP, Patowary A, Sarkar M, Ali T, Gupta S, Garg M, Singh M, Purkanti R, Scaria V et al (2012) Proximity of H2A.Z containing nucleosome to the transcription start site influences gene expression levels in the mammalian liver and brain. Nucleic Acids Res 40: $8965-8978$

Blake DR, Vaseva AV, Hodge RG, Kline MP, Gilbert TSK, Tyagi V, Huang D, Whiten GC, Larson JE, Wang $X$ et al (2019) Application of a MYC degradation screen identifies sensitivity to CDK9 inhibitors in KRASmutant pancreatic cancer. Sci Signal 12: eaav7259

Bonisch C, Hake SB (2012) Histone H2A variants in nucleosomes and chromatin: more or less stable? Nucleic Acids Res 40: 10719-10741

Bönisch C, Schneider K, Pünzeler S, Wiedemann SM, Bielmeier C, Bocola M, Eberl HC, Kuegel W, Neumann J, Kremmer E et al (2012) H2A.Z.2.2 is an alternatively spliced histone H2A.Z variant that causes severe nucleosome destabilization. Nucleic Acids Res 40: 5951-5964

Boyarchuk E, Filipescu D, Vassias I, Cantaloube S, Almouzni G (2014) The histone variant composition of centromeres is controlled by the pericentric heterochromatin state during the cell cycle. J Cell Sci 127: $3347-3359$

Buenrostro JD, Giresi PG, Zaba LC, Chang HY, Greenleaf WJ (2013) Transposition of native chromatin for fast and sensitive epigenomic profiling of open chromatin, DNA-binding proteins and nucleosome position. Nat Methods 10: 1213-1218

Chevillard-Briet M, Quaranta M, Grézy A, Mattera L, Courilleau C, Philippe M, Mercier P, Corpet D, Lough J, Ueda T et al (2014) Interplay between chromatin-modifying enzymes controls colon cancer progression through Wnt signaling. Hum Mol Genet 23: 2120-2131

Clarkson MJ, Wells JR, Gibson F, Saint R, Tremethick DJ (1999) Regions of variant histone His2AvD required for Drosophila development. Nature 399: 694-697

Coller HA, Grandori C, Tamayo P, Colbert T, Lander ES, Eisenman RN, Golub TR (2000) Expression analysis with oligonucleotide microarrays reveals that MYC regulates genes involved in growth, cell cycle, signaling, and adhesion. Proc Natl Acad Sci U S A 97: 3260-3265

Coon JJ, Ueberheide B, Syka JE, Dryhurst DD, Ausio J, Shabanowitz J, Hunt DF (2005) Protein identification using sequential ion/ion reactions and tandem mass spectrometry. Proc Natl Acad Sci U S A 102: 9463-9468

Corces MR, Trevino AE, Hamilton EG, Greenside PG, Sinnott-Armstrong NA, Vesuna S, Satpathy AT, Rubin AJ, Montine KS, Wu B et al (2017) An improved ATAC-seq protocol reduces background and enables interrogation of frozen tissues. Nat Methods 14: 959-962 
Croft JA, Bridger JM, Boyle S, Perry P, Teague P, Bickmore WA (1999) Differences in the localization and morphology of chromosomes in the human nucleus. J Cell Biol 145: 1119-1131

van Daal A, Elgin SC (1992) A histone variant, H2AvD, is essential in Drosophila melanogaster. Mol Biol Cell 3: 593-602

Dalvai M, Fleury L, Bellucci L, Kocanova S, Bystricky K (2013) TIP48/Reptin and H2A.Z requirement for initiating chromatin remodeling in estrogenactivated transcription. PLoS Genet 9: e1003387

de Castro IJ, Budzak J, Di Giacinto ML, Ligammari L, Gokhan E, Spanos C, Moralli D, Richardson C, de las Heras JI, Salatino S et al (2017) Repo-Man/ PP1 regulates heterochromatin formation in interphase. Nat Commun 8: 14048

Draker R, Ng MK, Sarcinella E, Ignatchenko V, Kislinger T, Cheung P (2012) A combination of H2A.Z and $\mathrm{H} 4$ acetylation recruits Brd2 to chromatin during transcriptional activation. PLoS Genet 8: e1003047

Dryhurst D, Ishibashi T, Rose KL, Eirín-López JM, MCDonald D, Silva-Moreno B, Veldhoen N, Helbing CC, Hendzel MJ, Shabanowitz J et al (2009) Characterization of the histone H2A.Z-1 and H2A.Z-2 isoforms in vertebrates. BMC Biol 7: 86

Dunn CJ, Sarkar P, Bailey ER, Farris S, Zhao M, Ward JM, Dudek SM, Saha RN (2017) Histone hypervariants H2A.Z.1 and H2A.Z.2 play independent and context-specific roles in neuronal activity-induced transcription of Arc/ Arg3.1 and other immediate early genes. eNeuro 4: ENEURO.0040-17.2017

Faast R, Thonglairoam V, Schulz TC, Beall J, Wells JR, Taylor H, Matthaei K, Rathjen PD, Tremethick DJ, Lyons I (2001) Histone variant H2A.Z is required for early mammalian development. Curr Biol 11: 1183-1187

Fan JY, Rangasamy D, Luger K, Tremethick DJ (2004) H2A.Z alters the nucleosome surface to promote HP1alpha-mediated chromatin fiber folding. Mol Cell 16: 655-661

Filipescu D, Szenker E, Almouzni G (2013) Developmental roles of histone H3 variants and their chaperones. Trends Genet 29: 630-640

Finlan LE, Sproul D, Thomson I, Boyle S, Kerr E, Perry P, Ylstra B, Chubb JR, Bickmore WA (2008) Recruitment to the nuclear periphery can alter expression of genes in human cells. PLoS Genet 4: e1000039

Foltz DR, Jansen LE, Black BE, Bailey AO, Yates 3rd JR, Cleveland DW (2006) The human CENP-A centromeric nucleosome-associated complex. Nat Cell Biol 8: $458-469$

Gevry N, Chan HM, Laflamme L, Livingston DM, Gaudreau L (2007) p21 transcription is regulated by differential localization of histone H2A.Z. Genes Deu 21: 1869-1881

Giaimo BD, Ferrante F, Vallejo DM, Hein K, Gutierrez-Perez I, Nist A, Stiewe T, Mittler G, Herold S, Zimmermann T et al (2018) Histone variant H2A.Z deposition and acetylation directs the canonical Notch signaling response. Nucleic Acids Res 46: 8197-8215

Giaimo BD, Ferrante F, Herchenrother A, Hake SB, Borggrefe T (2019) The histone variant H2A.Z in gene regulation. Epigenetics Chromatin 12: 37

Greenberg RS, Long HK, Swigut T, Wysocka J (2019) Single amino acid change underlies distinct roles of H2A.Z subtypes in human syndrome. Cell 178: 1421-1436.e1424

Guillemette B, Bataille AR, Gevry N, Adam M, Blanchette M, Robert F, Gaudreau L (2005) Variant histone H2A.Z is globally localized to the promoters of inactive yeast genes and regulates nucleosome positioning. PLOS Biol 3: e384

Halley JE, Kaplan T, Wang AY, Kobor MS, Rine J (2010) Roles for H2A.Z and its acetylation in GAL1 transcription and gene induction, but not GAL1transcriptional memory. PLoS Biol 8: e1000401

Harrow J, Frankish A, Gonzalez Jm, Tapanari E, Diekhans M, Kokocinski F, Aken BI, Barrell D, Zadissa A, Searle S et al (2012) GENCODE: the reference human genome annotation for The ENCODE Project. Genome Res 22: $1760-1774$

Horikoshi N, Sato K, Shimada K, Arimura Y, Osakabe A, Tachiwana H, Hayashi-Takanaka Y, Iwasaki W, Kagawa W, Harata M et al (2013) Structural polymorphism in the L1 loop regions of human H2A.Z.1 and H2A.Z.2. Acta Crystallogr D Biol Crystallogr 69: 2431-2439

Hua S, Kallen CB, Dhar R, Baquero MT, Mason CE, Russell BA, Shah PK, Liu J, Khramtsov A, Tretiakova MS et al (2008) Genomic analysis of estrogen cascade reveals histone variant H2A.Z associated with breast cancer progression. Mol Syst Biol 4: 188

Inoue A, Hyle J, Lechner MS, Lahti JM (2008) Perturbation of HP1 localization and chromatin binding ability causes defects in sister-chromatid cohesion. Mutat Res 657: $48-55$

Jackson JD, Gorovsky MA (2000) Histone H2A.Z has a conserved function that is distinct from that of the major H2A sequence variants. Nucleic Acids Res 28: $3811-3816$

Kang J, Chaudhary J, Dong H, Kim S, Brautigam CA, Yu H (2011) Mitotic centromeric targeting of $\mathrm{HPI}$ and its binding to $\mathrm{Sgol}$ are dispensable for sister-chromatid cohesion in human cells. Mol Biol Cell 22: $1181-1190$

Kelly AE, Ghenoiu C, Xue JZ, Zierhut C, Kimura H, Funabiki H (2010) Survivin reads phosphorylated histone $\mathrm{H} 3$ threonine 3 to activate the mitotic kinase Aurora B. Science 330: 235-239

Kelly RD, Cowley SM (2013) The physiological roles of histone deacetylase (HDAC) 1 and 2: complex co-stars with multiple leading parts. Biochem Soc Trans 41: 741-749

Kim D, Pertea G, Trapnell C, Pimentel H, Kelley R, Salzberg SL (2013a) TopHat2: accurate alignment of transcriptomes in the presence of insertions, deletions and gene fusions. Cenome Biol 14: R36

Kim K, Punj V, Choi J, Heo K, Kim JM, Laird PW, An W (2013b) Gene dysregulation by histone variant H2A.Z in bladder cancer. Epigenetics Chromatin 6: 34

Lamaa A, Humbert J, Aguirrebengoa M, Cheng X, Nicolas E, Cote J, Trouche D (2020) Integrated analysis of H2A.Z isoforms function reveals a complex interplay in gene regulation. Elife 9: e53375

Ledesma-Fernandez E, Thorpe PH (2015) Fluorescent foci quantitation for high-throughput analysis. J Biol Methods 2: e22

Li B, Pattenden SG, Lee D, Gutierrez J, Chen J, Seidel C, Gerton J, Workman JL (2005) Preferential occupancy of histone variant H2AZ at inactive promoters influences local histone modifications and chromatin remodeling. Proc Natl Acad Sci U S A 102: 18385-18390

Li H, Durbin R (2009) Fast and accurate short read alignment with BurrowsWheeler transform. Bioinformatics 25: 1754-1760

Li H, Handsaker B, Wysoker A, Fennell T, Ruan J, Homer N, Marth G, Abecasis G, Durbin R, 1000 Genome Project Data Processing Subgroup (2009) The sequence alignment/map format and SAMtools. Bioinformatics 25 : $2078-2079$

Love MI, Huber W, Anders S (2014) Moderated estimation of fold change and dispersion for RNA-seq data with DESeq2. Genome Biol 15: 550

Manzione MG, Rombouts J, Steklov M, Pasquali L, Sablina A, Gelens L, Qian J, Bollen M (2020) Co-regulation of the antagonistic RepoMan:Aurora-B pair in proliferating cells. Mol Biol Cell 31: 419-438

Matsuda R, Hori T, Kitamura H, Takeuchi K, Fukagawa T, Harata M (2010) Identification and characterization of the two isoforms of the vertebrate H2A.Z histone variant. Nucleic Acids Res 38: 4263-4273

McGuinness BE, Hirota T, Kudo NR, Peters JM, Nasmyth K (2005) Shugoshin prevents dissociation of cohesin from centromeres during mitosis in vertebrate cells. PLoS Biol 3: e86 
Mejia JE, Alazami A, Willmott A, Marschall P, Levy E, Earnshaw WC, Larin Z (2002) Efficiency of de novo centromere formation in human artificial chromosomes. Genomics 79: 297-304

Millar CB, Xu F, Zhang K, Grunstein M (2006) Acetylation of H2AZ Lys 14 is associated with genome-wide gene activity in yeast. Genes Deu 20: $711-722$

Naumova N, Imakaev M, Fudenberg G, Zhan Y, Lajoie BR, Mirny LA, Dekker ] (2013) Organization of the mitotic chromosome. Science 342: 948-953

Nonaka N, Kitajima T, Yokobayashi S, Xiao G, Yamamoto M, Grewal SI, Watanabe $Y$ (2002) Recruitment of cohesin to heterochromatic regions by Swi6/HP1 in fission yeast. Nat Cell Biol 4: 89-93

Perera D, Taylor SS (2010) Sgo1 establishes the centromeric cohesion protection mechanism in $\mathrm{G} 2$ before subsequent Bub1-dependent recruitment in mitosis. J Cell Sci 123: 653-659

Procida T, Friedrich T, Jack APM, Peritore M, Bonisch C, Eberl HC, Daus N, Kletenkov K, Nist A, Stiewe T et al (2021) JAZF1, a novel p400/TIP60/NuA4 complex member, regulates H2A.Z acetylation at regulatory regions. Int $\mathrm{J}$ Mol Sci 22: 678

Pünzeler S, Link S, Wagner G, Keilhauer EC, Kronbeck N, Spitzer RMM, Leidescher S, Markaki Y, Mentele E, Regnard C et al (2017) Multivalent binding of PWWP2A to H2A.Z regulates mitosis and neural crest differentiation. EMBO J 36: 2263-2279

Qian Y, Chen X (2010) Tumor suppression by p53: making cells senescent. Histol Histopathol 25: 515-526

Raisner RM, Hartley PD, Meneghini MD, Bao MZ, Liu CL, Schreiber SL, Rando OJ, Madhani HD (2005) Histone variant H2A.Z marks the 5' ends of both active and inactive genes in euchromatin. Cell 123: 233-248

Rangasamy D, Berven L, Ridgway P, Tremethick DJ (2003) Pericentric heterochromatin becomes enriched with H2A.Z during early mammalian development. EMBO J 22: 1599-1607

Rispal J, Baron L, Beaulieu JF, Chevillard-Briet M, Trouche D, Escaffit F (2019) The H2A.Z histone variant integrates Wnt signaling in intestinal epithelial homeostasis. Nat Commun 10: 1827

Ruppert JG, Samejima K, Platani M, Molina O, Kimura H, Jeyaprakash AA, Ohta S, Earnshaw WC (2018) HPlalpha targets the chromosomal passenger complex for activation at heterochromatin before mitotic entry. EMBO J 37: e97677

Ryan DP, Tremethick DJ (2018) The interplay between H2A.Z and H3K9 methylation in regulating HP1alpha binding to linker histone-containing chromatin. Nucleic Acids Res 46: 9353-9366

Skene PJ, Henikoff S (2013) Histone variants in pluripotency and disease. Development 140: $2513-2524$

Slupianek A, Yerrum S, Safadi FF, Monroy MA (2010) The chromatin remodeling factor SRCAP modulates expression of prostate specific antigen and cellular proliferation in prostate cancer cells. J Cell Physiol 224: $369-375$
Trapnell C, Williams BA, Pertea G, Mortazavi A, Kwan G, van Baren MJ, Salzberg SL, Wold BJ, Pachter L (2010) Transcript assembly and quantification by RNA-Seq reveals unannotated transcripts and isoform switching during cell differentiation. Nat Biotechnol 28: 511-515

Turinetto V, Giachino C (2015) Histone variants as emerging regulators of embryonic stem cell identity. Epigenetics 10: $563-573$

Uxa S, Bernhart SH, Mages CFS, Fischer M, Kohler R, Hoffmann S, Stadler PF, Engeland K, Muller GA (2019) DREAM and RB cooperate to induce gene repression and cell-cycle arrest in response to p53 activation. Nucleic Acids Res 47: 9087-9103

Vagnarelli P, Hudson DF, Ribeiro SA, Trinkle-Mulcahy L, Spence JM, Lai F, Farr C], Lamond Al, Earnshaw WC (2006) Condensin and Repo-Man-PP1 cooperate in the regulation of chromosome architecture during mitosis. Nat Cell Biol 8: 1133-1142

Vagnarelli P, Ribeiro S, Sennels L, Sanchez-Pulido L, de Lima AF, Verheyen T, Kelly DA, Ponting CP, Rappsilber J, Earnshaw WC (2011) Repo-Man coordinates chromosomal reorganization with nuclear envelope reassembly during mitotic exit. Deu Cell 21: $328-342$

Vardabasso C, Gaspar-Maia A, Hasson D, Pünzeler S, Valle-Garcia D, Straub T, Keilhauer E, Strub T, Dong J, Panda T et al (2015) Histone variant H2A.Z.2 mediates proliferation and drug sensitivity of malignant melanoma. Mol Cell 59: $75-88$

Wang F, Dai J, Daum JR, Niedzialkowska E, Banerjee B, Stukenberg PT, Gorbsky GJ, Higgins JM (2010) Histone H3 Thr-3 phosphorylation by Haspin positions Aurora B at centromeres in mitosis. Science 330: $231-235$

West MH, Bonner WM (1980) Histone 2A, a heteromorphous family of eight protein species. Biochemistry 19: $3238-3245$

Whittle CM, McClinic KN, Ercan S, Zhang X, Green RD, Kelly WG, Lieb JD (2008) The genomic distribution and function of histone variant HTZ-1 during C. elegans embryogenesis. PLoS Genet 4: e1000187

Wratting D, Thistlethwaite A, Harris M, Zeef LA, Millar CB (2012) A conserved function for the H2A.Z C terminus. J Biol Chem 287: $19148-19157$

Yamagishi Y, Honda T, Tanno Y, Watanabe Y (2010) Two histone marks establish the inner centromere and chromosome bi-orientation. Science 330: $239-243$

Yoon MH, Kang SM, Lee SJ, Woo TG, Oh AY, Park S, Ha NC, Park BJ (2019) p53 induces senescence through Lamin A/C stabilization-mediated nuclear deformation. Cell Death Dis 10: 107

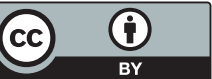

License: This is an open access article under the terms of the Creative Commons Attribution License, which permits use, distribution and reproduction in any medium, provided the original work is properly cited. 